\title{
Unresonant interaction of laser beams with microdroplets
}

\author{
M. L. Pascu \\ mihai.pascu@inflpr.ro \\ G. V. Popescu
}

\section{M. Ticos}

\section{R. Andrei}

\author{
National Institute for Laser, Plasma and Radiation Physics, Laser Department, Magurele, 077125, Ro- \\ mania \\ National Institute for Laser, Plasma and Radiation Physics, Laser Department, Magurele, 077125, Ro- \\ mania \\ National Institute for Laser, Plasma and Radiation Physics, Laser Department, Magurele, 077125, Ro- \\ mania \\ National Institute for Laser, Plasma and Radiation Physics, Laser Department, Magurele, 077125, Ro- \\ mania
}

The interaction of distilled water droplets (volumes of 3-4 $\mu \mathrm{l}$ ) with pulsed laser beams emitted at $532 \mathrm{~nm}$ is described. At $532 \mathrm{~nm}$ the distilled water absorption is very low and the interaction of a water droplet with the laser radiation is dominated by unresonant phenomena. In this case, following the collision of the laser beam with a droplet in suspended position in air, its deformations and mechanical vibrations are produced. The conditions in which the droplets lose material as a consequence of the impact with laser beams are explored. The effects produced on the droplet were studied pulse by pulse and depend on: droplet's content, beam wavelength, power and focusing, irradiation geometry and adhesion of the droplet to the capillary on which it is suspended. The laser pulses energies were varied in four steps: $0.25 \mathrm{~mJ}, 0.4 \mathrm{~mJ}, 0.7 \mathrm{~mJ}$ and $1 \mathrm{~mJ}$. The laser pulse full time width at half maximum was $5 \mathrm{~ns}$ and the typical beam waist diameter on the droplet was $90 \mu \mathrm{m}$; the beam had a relatively low divergence around the focus point. The droplet's shapes evolution is visualised by recordings performed at $10 \mathrm{kframes} / \mathrm{second}$. Following a droplet interaction with the laser beam one may also produce at a controlled moment in time, nanodroplets propagating at high (probably supersonic) speeds and microdroplets propagating at slower speeds. One may also produce suspended droplets of smaller dimensions than the initial one as well as micro/nano gas bubbles in the suspended droplet's material/volume. In a second set of experiments the behaviour of the microdroplets of Rhodamine 6G in distilled water was recorded at high speed, at resonant interaction with similar laser pulses, and at the same power levels. The phenomena considering that the droplets contents are Newtonian liquids produced at interaction with the laser beams, are discussed.

[DOI: http://dx.doi.org/10.2971/jeos.2012.12001]

Keywords: microfluidics, laser, unresonant/resonant interaction, optical radiation pressure

\section{INTRODUCTION}

The need to use millimetre-, micro- and even nano-droplets in technological and biomedical applications triggered research related to new methods for their production and more accurate characterisation [1]. The optical and laser methods used to study droplets are in privileged position, constituting a significant part of a relatively new and dynamic field: optofluidics $[2,3]$.

Literature reports show results about the laser beam interaction with droplets that contain single liquids or mixtures of immiscible materials produced in different surrounding environments (which may be either liquids or air/gases) [4, 5].

The most studied droplets (either quasi- or hemi-spherical) are placed on hydrophobic or superhydrophobic (or even oleophobic or superoleophobic) transparent surfaces. The interaction with the optical radiation takes place by transmission of the laser beams through the support surface [6]. In other cases the droplets are produced and fixed or moved within microchannel structures that are designed for their ma- nipulation by light in single- or two - phase flows, in view of their further use in optical chips $[7,8]$.

Results are reported on microdroplets free falling in air, levitated or embedded in other liquid and are related to the emission by the droplets of fluorescence or even lased radiation at pumping with laser beams [9]-[11]. An important role in the droplets behaviour under optical radiation is played by their components (materials to study, solvents in which these are dissolved, dyes, fluorophores), the medium surrounding the droplet and the structure of it (the droplet may be simple or may contain a core and one or more covering thin layers of immiscible materials [1]). Among solvents, the most used are the alcohols, particularly the ethyl alcohol, but polymeric materials are also employed in some cases [10]. Water is another material which is commonly used for droplets' generation since its optical, physico-chemical and fluidic properties are relatively well known. However, many of the water properties behaviours or responses at the interaction with laser beams remain unknown, and this justifies the studies of its interac- 


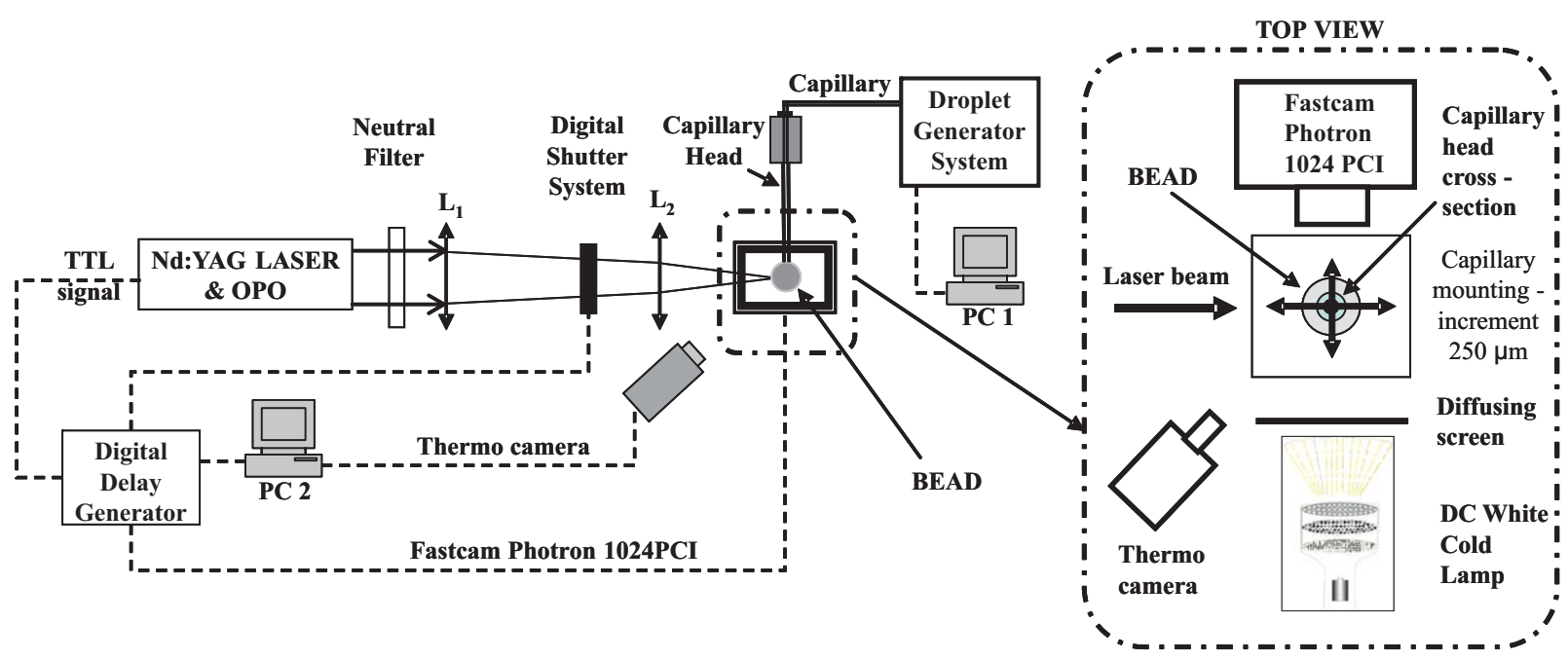

FIG. 1 Overall scheme of the experimental set-up.

tion with the coherent optical radiation in bulk $[7,12,13]$, or under the shape of droplets as it is reported in this paper.

The interaction of the droplets with laser beams focused on them may be resonant or unresonant. In this paper the term "resonant" is used with its meaning in optical spectroscopy: the radiation is absorbed by the molecules in the droplets and these molecules become excited on singlet states by absorption of one or more photons of pumping radiation. If the concentration of the absorbent is optimal with respect to the intensity of the incoming laser beam i.e. with the number of photons in the light pulse, the photons may be absorbed in the first layers of liquid in the droplet and they cannot propagate further in it. A possible consequence of the excitation of the molecules on excited singlet states may be the heating of the droplet due to nonradiative transfers of molecules within the singlet states or due to the intersystem (singlet-triplet) crossing.

The term unresonant is used in this paper when the wavelength of the laser beam is chosen so that it is not absorbed by the droplet's material. This takes place usually in single material droplets as is the case of distilled water where the effects of the light pressure, electrostrictive forces originating in the laser beam, possible thermal effects on the droplet, dominate the interaction scene [14].

The use of pendant droplets that are hanging on a capillary, allows one to study the individual interaction of liquid droplets with laser beams in different surrounding media assuring to the droplets the conditions to freely modify their shapes but keeping them, within some limits, connected to the capillary.

In this paper the interaction of distilled water droplets having volumes in the microliter range with pulsed laser beams emitted at $532 \mathrm{~nm}$ is described. At this wavelength the distilled water absorption is very low and the interaction of the microdroplets with the laser beam is dominated by unresonant phenomena. The laser pulses energies were modified in several steps and the pulse full time width at half maximum (FTWHM) was $5 \mathrm{~ns}$; the beam waist on the droplet was around $90 \mu \mathrm{m}$. Following the impact of the pendant droplets with laser beams of increasing energies, their deformations and mechanical vibrations were produced. The conditions in which the droplets lost material as a consequence of the impact with the laser beam of higher energy were also explored. The effects produced on single droplets were studied pulse by pulse and depended on droplets content, laser beam wavelength, power and focusing conditions, irradiation geometry and interaction of the droplets with the capillary on which they were hanging. The droplets and the modification/evolution of their shapes were visualised by high speed recording camera. Following a droplet interaction with laser beams nanodroplets were produced propagating at high speed and microdroplets propagating at slower speeds. Pendant droplets of smaller dimensions than the initial ones as well as micro/nano gas bubbles within their volume were also produced. The generation of nanodroplets that are free propagating in air/gas is of interest for nanoscience, nanotechnology and nanomedicine when it comes to use nanodroplets of materials freely propagating in gas media and falling on surfaces whose properties may be locally modified in a controlled manner.

A second kind of experiment was the high speed recording of the behaviour of the microdroplets of Rhodamine 6G (R6G) in distilled water at resonant interaction with the same types of laser pulses. The results obtained in this section are of outmost interest, in authors opinion, since they suggest new ways to obtain combined effects on droplets at interaction with laser beams when the absorption level of the laser beam in the droplet is controlled (by adjusting the absorbent concentration) from non-absorbing to high absorption.

\section{EXPERIMENTAL SET-UP}

The general structure of the experimental set-up is given in Figure 1. The droplets were generated in hanging/pendant position in open air, one by one, using a computer controlled (PC1) Droplet Generator System (MICROLAB 500 Dual Syringe Diluter, Hamilton) which produces droplets of maximal volume $50 \mu \mathrm{l}$; the resolution of the system is $0.05 \%$ of the utilised syringe volume, i.e. $0.0025 \mu \mathrm{l}$ at the maximal volume, the accuracy in generating a volume is $\pm 1 \%$ and the precision 


\begin{tabular}{|c|c|c|c|c|c|c|c|}
\hline \multirow{2}{*}{ Volume ( $\mu 1)$} & \multirow{2}{*}{ Focus position } & \multicolumn{2}{|c|}{ Substance } & \multicolumn{4}{|c|}{ Energy (mJ) } \\
\hline & & Water & R6G & E1 & E2 & E3 & E4 \\
\hline \multirow{3}{*}{3.0} & 1 & $X$ & & $X$ & $X$ & $X$ & $X$ \\
\hline & 2 & $X$ & & $\mathrm{X}$ & $\mathrm{X}$ & $X$ & $X$ \\
\hline & 3 & $X$ & & $X$ & $X$ & $X$ & $X$ \\
\hline \multirow{8}{*}{3.5} & 1 & $X$ & & $X$ & $x$ & $X$ & $X$ \\
\hline & 2 & $X$ & & $\mathrm{X}$ & $\mathrm{X}$ & $X$ & $X$ \\
\hline & 3 & $\bar{X}$ & & $X$ & $x$ & $X$ & $X$ \\
\hline & 4 & $\bar{X}$ & & $X$ & $X$ & $X$ & $X$ \\
\hline & 5 & $X$ & & $X$ & $X$ & $X$ & $X$ \\
\hline & 6 & $X$ & & $X$ & $X$ & $X$ & $X$ \\
\hline & 7 & $X$ & & $X$ & $X$ & $X$ & $X$ \\
\hline & 8 & $X$ & & $X$ & $X$ & $X$ & $X$ \\
\hline \multirow{3}{*}{4.0} & 1 & $X$ & & $x$ & $x$ & $X$ & $X$ \\
\hline & 2 & $X$ & & $X$ & $\mathrm{X}$ & $X$ & $X$ \\
\hline & 3 & $X$ & & $X$ & $X$ & $X$ & $X$ \\
\hline \multirow{3}{*}{3.0} & 1 & & $X$ & $X$ & $X$ & $X$ & $X$ \\
\hline & 2 & & $\mathbf{X}$ & $X$ & $X$ & $X$ & $X$ \\
\hline & 3 & & $\mathbf{X}$ & $\mathbf{X}$ & $\mathbf{X}$ & $X$ & $X$ \\
\hline
\end{tabular}

TABLE 1 Droplets exposure to laser beams at various volumes and contents, beam energies and focus positions.

is $\pm 0.2 \%$. The capillary material was hydrophobic so that the droplet was tangent to the capillary tip and its material did not adhere to the capillary inner or outer surface. Droplets with three volumes were generated: $3,3.5$ and $4 \mu 1$ (computed diameters of $1.79,1.88,1.97 \mathrm{~mm}$, respectively). In our studies distilled water was used (Barloworld, W4000); for resonant interaction, the droplets contained solutions of R6G in distilled water, at $10^{-3} \mathrm{M}$.

A Nd:YAG laser (Continuum Surelite) coupled with an OPO (Continuum Panther) were used, which emit by SHG at $532 \mathrm{~nm}$, pulses of laser radiation of $5 \mathrm{~ns}$ FTWHM and repetition rate $10 \mathrm{pps}$. Four steps of the average energy per laser pulse were used: $0.25,0.4,0.7$ and $1 \mathrm{~mJ}$. The laser beam energy transmitted to the droplet was controlled with a Neutral Filter which besides filtering the $532 \mathrm{~nm}$, cut-off the most part of the remaining fundamental radiation at $1.064 \mu \mathrm{m}$. The beam was focussed on the droplet, using a lens doublet $\mathrm{L}_{1}-\mathrm{L}_{2}$ out of which $\mathrm{L}_{2}$ is a $20 \mathrm{x}$ microscope objective with a $0.09 \%$ reflectivity at $532 \mathrm{~nm}$; the focus was placed at $8 \mathrm{~mm}$ after $\mathrm{L}_{2}$ and it had a cross section of minimum $90 \mu \mathrm{m}$ diameter; the beam waist around the focus was such that the $90 \mu \mathrm{m}$ diameter remained constant over $250 \mu \mathrm{m}$ along the propagation direction of the laser beam, i.e. at least $12 \%$ from the droplet diameter. The droplet was screened using a thermoisolating box to avoid air currents around it and to minimise the water vaporisation and the heat exchanges with the surrounding air/atmosphere.

The evolution of the droplet was shown in real time on PC3 using dedicated software and was recorded using a FASTCAM PHOTRON 1024 PCI camera at a speed of 10 kiloframes/second; the maximum resolution of $1024 \times 1024$ pixels was only possible at 1000 frames/second. In order to obtain a better image contrast, a diffusing screen illuminated from behind by a DC supplied white cold lamp, opposite to the camera, was mounted so that the image contrast was better without heating the droplet (Figure 1, TOP VIEW).
The capillary outer diameter was $510 \mu \mathrm{m}$ and the inner diameter was $210 \mu \mathrm{m}$. The capillary could be finely moved, resulting in a change of the droplet's vertical position and the translation in the horizontal plane along the laser beam and perpendicular to it with an increment of $250 \mu \mathrm{m}$.

The droplet temperature was monitored (PC2) with an accuracy of $\pm 2{ }^{\circ} \mathrm{C}$ using a thermocamera ThermaCAM E45 (FLIR) which had a thermal sensitivity of $0.1^{\circ} \mathrm{C}$ at $25^{\circ} \mathrm{C}$ in the spectral range 7.5 to $13 \mu \mathrm{m}$ (Figure 1 ).

The laser beam access to the droplet was controlled by the Digital Shutter which was synchronised with the laser pulse generator and the fast camera (PC3) via a Digital Delay Generator. The camera was set so that for each shown picture the shutter was open for the whole period of $100 \mu \mathrm{s}$.

\section{RESULTS}

The measurements of unresonant interaction were performed on distilled water droplets by exposing them to $532 \mathrm{~nm}$ laser beams; the water absorption is very low, the absorption coefficient being $\sim 0.05 \mathrm{~m}^{-1}$, and one may consider that the absorption of the beam in the volume of the droplets is negligible $[15,16]$. The laser beam was focused at different positions on the droplet surface or within its volume, as shown in Figure 2 and Table 1. For the droplets that contained only distilled water the whole range of volumes was utilised. The droplet content for resonant interaction was R6G in distilled water and the droplet's volume was $3.0 \mu \mathrm{l}$.

On the other hand, the lens doublet $\mathrm{L}_{1}-\mathrm{L}_{2}$ produced a laser beam with a low divergence. The beam waist area along the interval A - B in Figure 3 is around $6350 \mu \mathrm{m}^{2}$ and at approximately $1 \mathrm{~mm}$ before or after the focus it is about one order of magnitude larger. Although the $1.064 \mu \mathrm{m}$ and 532nm beams are single mode, the intensity distribution at waist is neither 

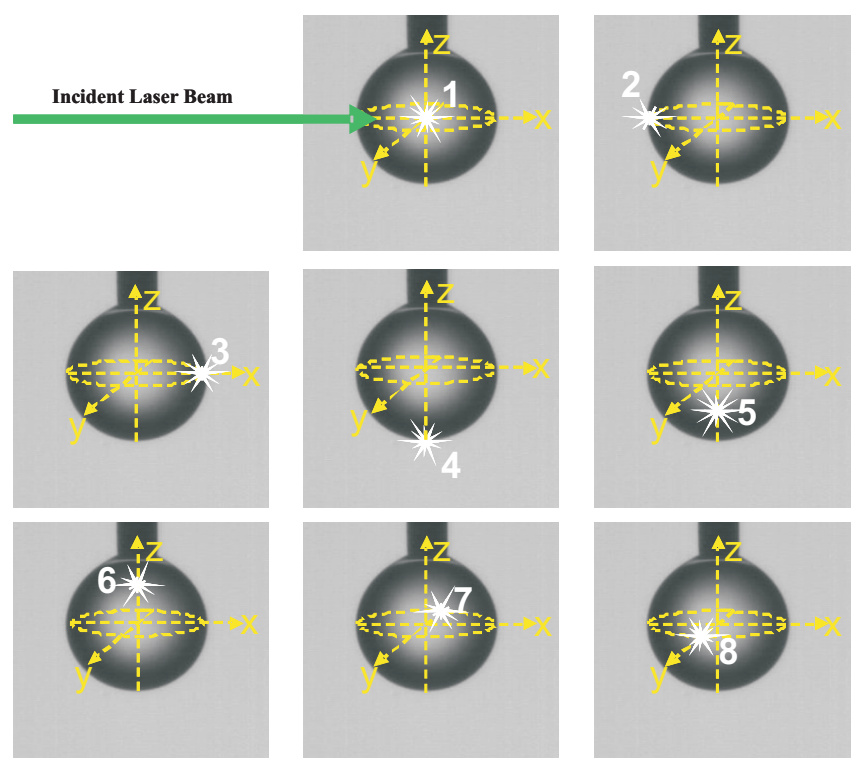

FIG. 2 Position of the laser beam focus on the droplet.

(I)
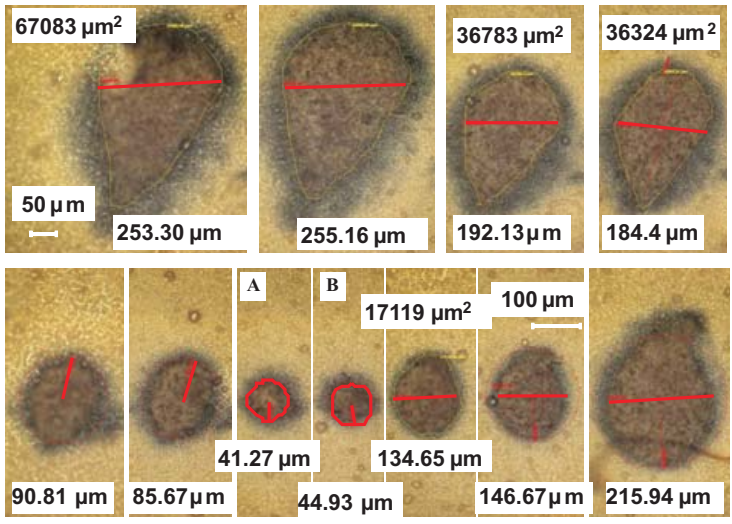

(II)

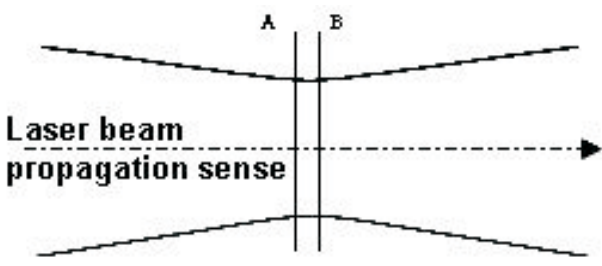

FIG. 3 Sections of the laser beam evidenced on sensitive paper. (I) Dimensions of the cross sections measured from left to right in the sense: laser - zone after the droplet; in some of the images the area of the beam waist is given in $\mu \mathrm{m}^{2}$; (II) Laser beam longitudinal section. The distance between two successive sections is $250 \mu \mathrm{m}$. The sections A and B in (I) and (II) represent the waist area. The pictures are taken with a Zeiss microscope, Axio Imager 2.

Gaussian, nor constant as shown in Figures 3 and 4. In Figure 4(a) the 2D distribution of the laser beam intensity at waist is shown; Figure 4(b) shows the 3D intensity distribution corresponding to the $2 \mathrm{D}$ image in the same conditions. It results that the laser beam intensity is not maximal on its optical axis, but in an off-axis position and that actually there is an intensity distribution in the cross section containing two unequal, but relatively intense peaks. This is confirmed by the images in Figure $3(I)$ which also show an elongation of the spot on vertical and the waist intensity distribution. The laser beam longitudinal section in Figure 3 (II) shows that the waist is constant for more than $250 \mu \mathrm{m}$; this is responsible for avoid-

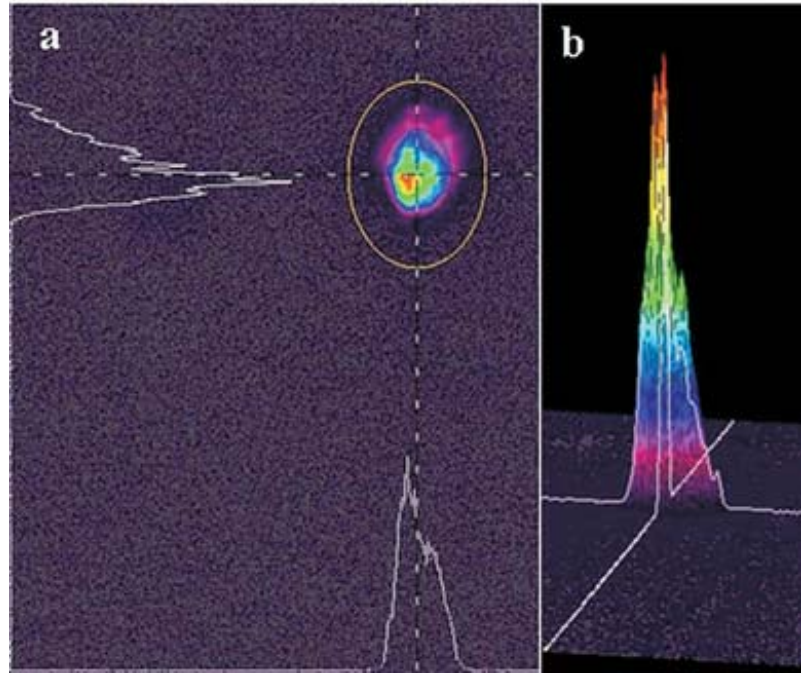

FIG. 4 The laser beam intensity in the focus, measured with SPIRICON, LW 230 Laser Beam profiler. The incident beam filtering allowed only $10^{-7}$ of the intensity to fall on the sensitive head. a. 2D distribution; b. 3D distribution corresponding to a.

\begin{tabular}{|c|c|c|c|}
\hline $\begin{array}{c}\text { Energy } \\
(\mathrm{mJ})\end{array}$ & $\begin{array}{c}\text { Power } \\
(\mathrm{KW})\end{array}$ & $\begin{array}{c}\text { Energy density } \\
\left(\mathrm{J} / \mathrm{cm}^{2}\right)\end{array}$ & $\begin{array}{c}\text { Power density } \\
\left(\mathrm{GW} / \mathrm{cm}^{2}\right)\end{array}$ \\
\hline 0.25 & 50 & 4.31 & 0.86 \\
\hline 0.4 & 80 & 6.89 & 1.38 \\
\hline 0.7 & 140 & 12.06 & 2.41 \\
\hline 1.0 & 200 & 17.22 & 3.44 \\
\hline
\end{tabular}

TABLE 2 Laser beam energy/power and beam energy/power density in the focus.

ing the breakdown at the contact of the laser beam with the droplet's surface, because conditions like those mentioned in [9] for instance, are not met. These laser beam characteristics have important consequences on the unresonant effects produced on the droplet when particular positions of the focus with respect to the droplet surface and centre are set. In the following, a synthesis of the results on the unresonant interaction are presented.

The correspondence between the beam energy in the focus and the energy and power densities on a droplet is presented in Table 2.

The results are displayed for each case as a movie and as a series of selected pictures extracted from the movie and associated with it. Each picture or frame contains light received by the camera during $100 \mu \mathrm{s}$. The synchronisation chain consisting of "laser pulse generator - digital delay generator - high speed camera - shutter" allows to record in the first frame the signals received during the last $10 \mu \mathrm{s}$ from the first $100 \mu \mathrm{s}$ after triggering of the measuring sequence. In other words the laser beam falls on the droplet at $90 \mu \mathrm{s}$ after the high speed camera starts to record the first frame. The frames taken during these $10 \mu \mathrm{s}$ are labelled $\left(^{*}\right)$ in the frames sequences. Each of the subsequent pictures contains the superposition of the droplet's images received during $100 \mu \mathrm{s}$.

We consider in this paper that the microdroplet contains a Newtonian liquid because our observations during the experiments have shown that the water droplets continue to have 


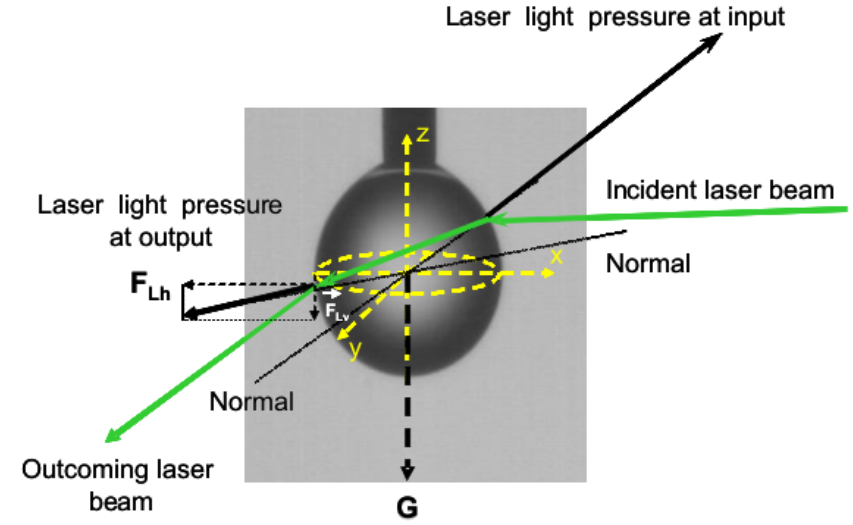

FIC. 5 Optical scheme of the laser beam propagation through the droplet and the light pressure produced by it.

the same fluid behaviour and properties during and after the interaction with the laser beams [17].

In Figure 5 the optical path of the laser beam in the droplet is drawn. Once fallen on the droplet the incoming beam is refracted at the surface of it twice: at the input and at the output. Along the optical path, the laser beam applies a light pressure on the droplet at the interface with the air, so that the pressure is always perpendicular to the droplet surface and its direction is from the water (higher refractive index) towards air (lower refractive index) [7, 18, 19]. This takes place regardless the incidence angle of the beam on the droplet at input, respectively output and of the position of the incidence points of the laser beam on the droplet surface.

In this way, the laser light pressure applied on the hanging droplet, which is with a good approximation spherical, is always oriented along the radius of curvature of the droplet at the contact points. Moreover, at input, the pressure will have the direction oriented more or less contrary-wise with respect to the laser beam; at output from the droplet the pressure will be also oriented from the droplet towards the air, i.e. more or less in the same sense with the laser beam (Figure 5). This is obvious if the laser beam is sent on the droplet in the equatorial plane of it; in this case the deformation of the droplet, and possibly the loss of material will be produced by $\mathbf{F}_{L h}$, only, in interaction with the viscosity forces of the material, the droplet surface tension and other possible effects on it such as the electrostriction $[12,20]$ and the thermal gradients.

\subsection{Unresonant interaction}

The effects of the unresonant interaction are presented in movies and associated sets of pictures within which the time intervals specified between some of the frames indicate that frames were skipped to better identify the differences between the adjacent pictures. Each movie scan can be accessed if clicking on the frames labelled $\left(^{*}\right)$.

\section{1 .1}

Interaction of the laser beam sent in the equatorial plane of the droplet and focused in its geometrical centre (position 1 in Figure 2).
In Figure 6 the evolution of an individual droplet of $3 \mu 1$ volume after interacting with a single laser pulse of $0.4 \mathrm{~mJ}$ is shown. The figure describes phenomena in the same category as those introduced in [21, 22]. From Figure 6, frame 1 (see also frame 1 magnified 3 times), it results that emission of jets that may be constituted from nanodroplets is produced at the input of the laser beam in the droplet, faster than the emission at the output from it. The outer diameter of the capillary was considered as length reference and the exposure time was $100 \mu \mathrm{s}$. The jet propagation speeds were computed by measuring the length of the nanodroplets in the picture by using the time-of-flight technique. The speed of the jet at the input in the droplet is $13.6 \mathrm{~m} / \mathrm{s}$ and the jet speed at the output is $5.1 \mathrm{~m} / \mathrm{s}$.

From frame 2 it results that after more than $100 \mu \mathrm{s}$, a bulge is formed on the output surface in the point where the laser beam leaves the droplet. The approximate speed of the appearance of this deformation peak is about $1.5 \mathrm{~m} / \mathrm{s}$. The bulge is damped in more than $100 \mu \mathrm{s}$ as it results from frames 3 6 . The halo around the droplet in frame $\left(^{*}\right)$ is due to the total reflection of the $532 \mathrm{~nm}$ beam on droplet's external thin layer $[12,23,24]$.

In Figure 6, significant inhomogeneities are produced around the focus, which at $0.4 \mathrm{~mJ}$ energy generate low speed $(\sim$ $0.3 \mathrm{~m} / \mathrm{s}$ ) waves in the droplet. The waves are reflected by the capillary and the droplet surface leading to its deformation, and are damped afterwards (frames 7 to 11). Such inhomogeneities are also shown in Figure 7 where strong light scattering occurs on them. The scattered light puts pressure on the water in the droplet and may further generate the water jets in Figure 7 (see frames 1 to 5).

The measurements with the thermocamera show that the droplet's temperature does not increase during or after the interaction with the laser beam if its energy is between $0.25 \mathrm{~mJ}$ and $1 \mathrm{~mJ}$ [20]. The temperature remains constant within the limits of the measuring errors. So, the inhomogeneities produced within the droplet in the focus area may be generated by the electrostrictive effect produced by the laser beam on the water considered as a dielectric medium [25, 26]. It has been demonstrated that following the interaction of the laser beam which has an energy above a particular threshold an instability is produced within the liquid bulk that, formally, looks like an acoustic instability produced in plasma [26]. This is because the water/dielectric fluid flows under the interaction with the laser radiation from the volume where it has a lower energy density towards the volume where this density is higher; this process is converse with respect to the natural tendency of the water to flow from higher to lower pressures.

The inhomogeneities produce a stimulated light scattering, which, due to the fact that the water temperature remains constant, may be stimulated Brillouin scattering (SBS) and stimulated Rayleigh scattering induced by electrostriction. The spontaneous Brillouin and Rayleigh scattering are not dominant because the laser beam power is higher than the threshold that is needed to produce stimulated scattering phenomena. According to [27], the threshold beam power to produce SBS, is

$$
P_{t h}=\left(G_{t h} \cdot \lambda\right) / 2 g
$$



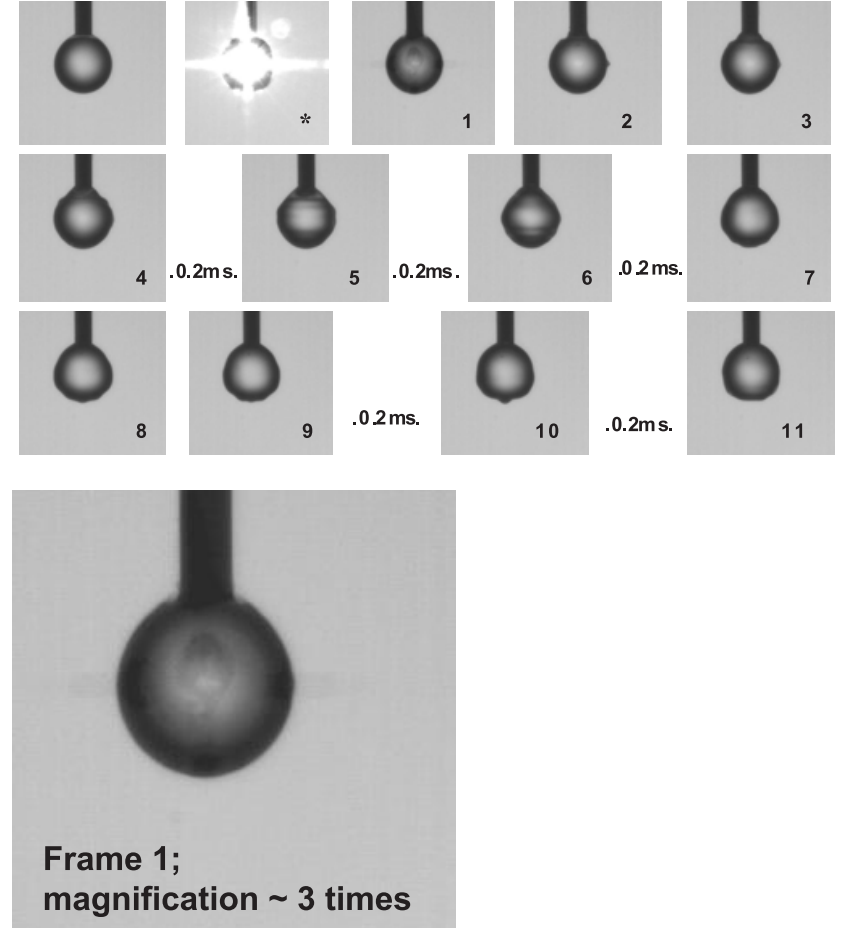

FIG. 6 Recording of the effects produced on the droplet by the laser beam sent in the equatorial plane and focused in its centre. Droplet volume $3 \mu \mathrm{l} ; \mathrm{E}_{2}=0.4 \mathrm{~mJ}$. The time intervals $0.2 \mathrm{~ms}$ between some pictures indicate that frames were skipped (in this case two) Movie-1 (4,9 MB).

where $G_{t h}=25$ is the gain parameter at the threshold, $\lambda$ the wavelength of the laser beam (532 nm) and the SBS gain factor $g=0.048 \mathrm{~m} / \mathrm{GW}$. The resulting $P_{t h}$ is $13.85 \mathrm{KW}$ that is lower than the laser beam powers used to obtain the effects shown in Figures 7 and 8. On the other hand, the light scattered as results indirectly from these figures is angularly distributed around the droplet, mainly in the forward direction of the laser beam. Normally, SBS takes place in bulk samples in the backward sense of the beam if the sample is long enough [26]. It seems that in the droplets this effect has lower intensity since their diameters are small $(\sim 2 \mathrm{~mm})$ and the most important part of light is scattered around the droplet in the $2 \pi$ forward volume. This result may confirm the electrostriction mechanism of light scattering by the instability produced in the droplet at focus; the mechanism is suggested for compressible fluids with extension to water droplets exposed to high energy laser beams weakly absorbed by the water [26].

The light scattered by the unhomogeneities induced in the droplet by the laser beam produces pressure on the water that is maximal where the light reaches the droplet surface and jets of water material as well as micro- and nano-droplets are expelled along the droplet radiuses in the respective points. In Figures 7 and 8 the jet at the incidence point on the droplet is emitted faster than the other jets, as it results from frames 1 in Figures 7 and 8. But this jet is rather due to the light pressure on the droplet than to the light scattering on unhomogeneities. Slower motions in the droplets volume are produced which lead in Figures 7 and 8 to the detachment of the droplets from the capillary, accompanied by emission in $4 \pi$ of micro- and nanodroplets that are not organised in jets and that may coalesce (see frames 2 to 9 in Figures 7 and 8).

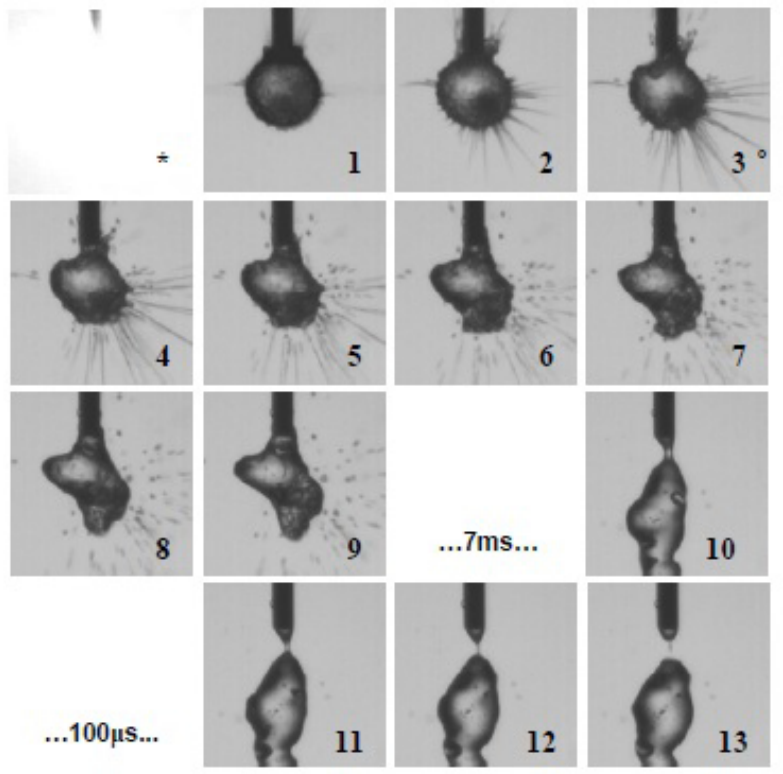

FIG. 7 Recording of the effects produced on the droplet by the laser beam sent in the equatorial plane and focused in its centre. Droplet volume $3 \mu \mathrm{l} ; \mathrm{E}_{4}=1 \mathrm{~mJ}$ Movie-2 $(4,9 \mathrm{MB})$.

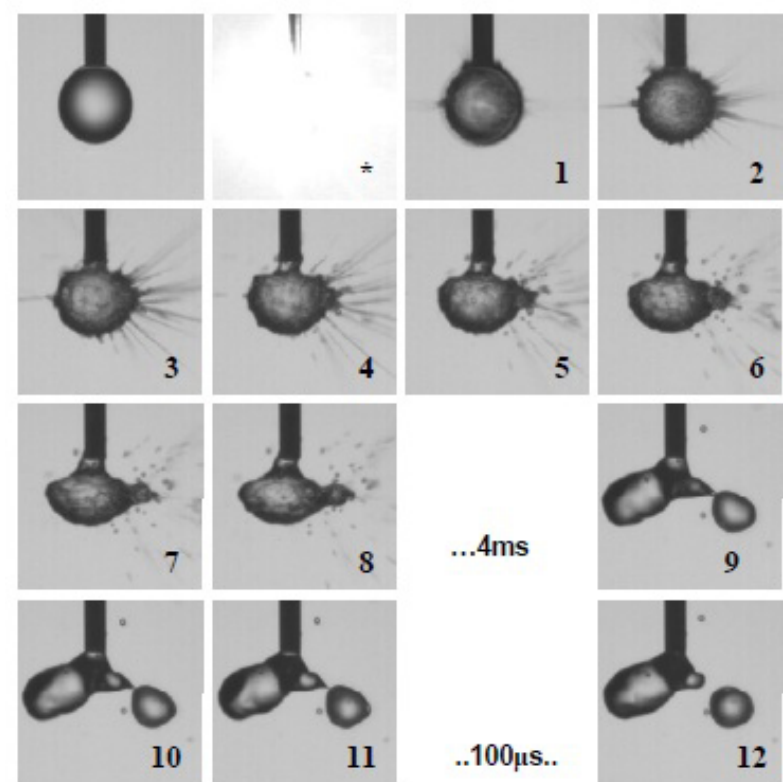

FIG. 8 Recording of the effects produced on the droplet by the laser beam sent in the equatorial plane and focused in its centre. Droplet volume $3.5 \mu \mathrm{l} ; \mathrm{E}_{4}=1 \mathrm{~mJ}$ Movie-3 $(4,9 \mathrm{MB})$.

In Figure 7 the time necessary for the droplet to leave the capillary is $8.3 \mathrm{~ms}$ and in Figure 8, $5.1 \mathrm{~ms}$

\section{1 .2}

Interaction of the laser beam incident on the droplet in the equatorial plane, on front surface (position 2 in Figure 2).

The effects are shown for droplet's volume of $3 \mu 1$ in Figures 9 to 11 , for $3.5 \mu \mathrm{l}$ volume in Figures 12 to 16 and for $4 \mu \mathrm{l}$ in Figures 17 to 19 . In all the cases, at the point of contact between the laser beam and the droplet, a water jet containing nanodroplets is emitted backward with respect to the laser ra- 


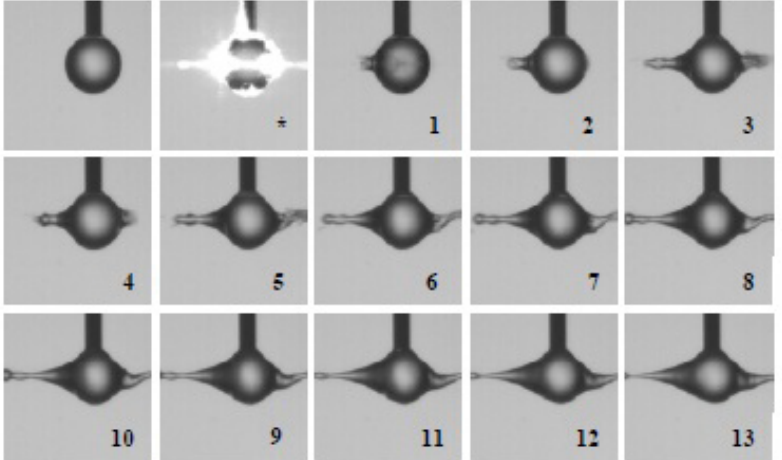

FIG. 9 Recording of the effects produced on the droplet by the laser beam sent in the equatorial plane and focused on the front face of it. Droplet volume $3 \mu \mathrm{l} ; \mathrm{E}_{1}=0.25 \mathrm{~mJ}$ Movie-4 (4,9 MB).

diation propagation direction; the same happens forward at the output from the droplet. As it results from the pictures labelled $\left({ }^{*}\right)$, the jets are evidenced by the light scattered on their components (see Section 3.1.1). If the scattering takes indeed place on the jets components, considering the speed of light in the droplet and in air, it results that the jets have a very high propagation speed, in the hypersonic range. At later times, in the backward direction lower speed jets are emitted that have, as a rule, a central cone structure circled by a liquid crown; the form of these jets strongly depends on the droplets dimensions, laser beam energy and the focusing conditions. They may be seen starting with frames 1 in Figures 9-19; their respective propagation speeds vary from $4.4 \mathrm{~m} / \mathrm{s}$ in Figure 17, to about $11 \mathrm{~m} / \mathrm{s}$ in Figure 12 and more than $20 \mathrm{~m} / \mathrm{s}$ in Figures 10-14, 18 and 19. In all figures starting with frame 2, the backward jets evolve with lower speeds and have different shapes function of the impact conditions, beam energy and droplets volumes.

Other liquid emissions take place at the output of the beam from the droplet (see, Figure 9 frame 3, Figure 10 frame 1 and Figure 11 frame 3) which are produced at latter stages (between $100 \mu \mathrm{s}$ and $400 \mu \mathrm{s}$ ) after the input of the laser beam in the droplet; the expulsion speeds vary from approximately $10.2 \mathrm{~m} / \mathrm{s}$ (Figure 9 frame 3) to $9.5 \mathrm{~m} / \mathrm{s}$ (Figure 10 frame 1), and $4.4 \mathrm{~m} / \mathrm{s}$ (Figure 11 frame 3 ). These speeds are lower than the speeds for the corresponding backward jets but in some cases (Figure 9) they may be higher due, probably, to the focusing geometry on the droplet.

From images it results that the position of the focus with respect to the droplet's surface is critical. This applies for all the volumes of the droplets and for all the power levels of the laser beam; a particular example is given in Figure 11.

The only difference is that the droplets generated by the computer controlled syringe system are different, i.e. one may have a slightly different diameter and volume from one droplet to another that according to our measurements may be of around $0.05 \mathrm{~mm}$.

Another reason for obtaining variable effects on the droplet may be the relatively small fluctuations of the laser beam energy, from one pulse to another, due to the jitter conditions,

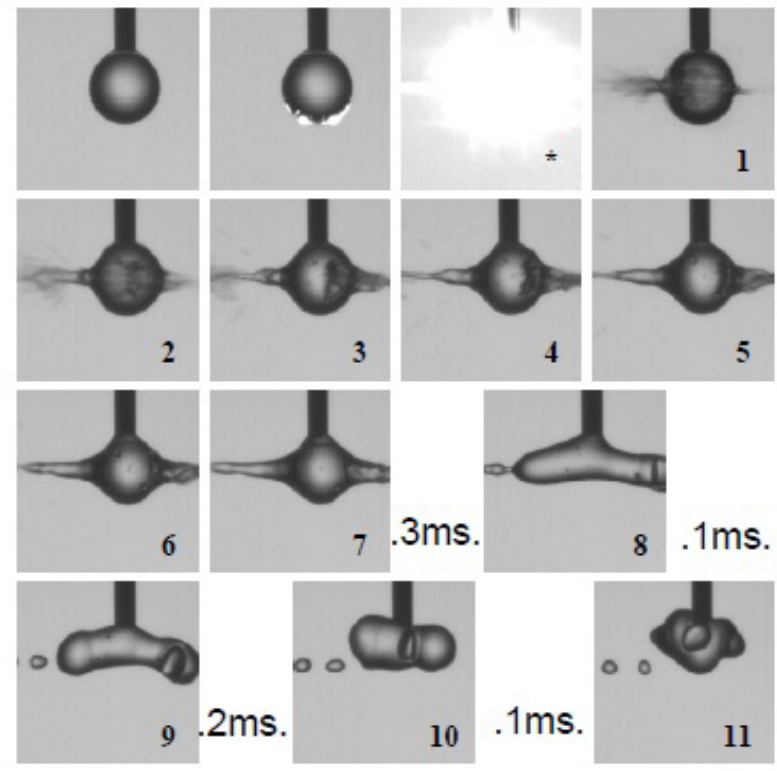

FIG. 10 Recording of the effects produced on the droplet by the laser beam sent in the equatorial plane and focused on the front face of the droplet. Droplet volume $3 \mu \mathrm{l}$; $E_{3}=0.7 \mathrm{~mJ}$ Movie-5 (4,9 MB).

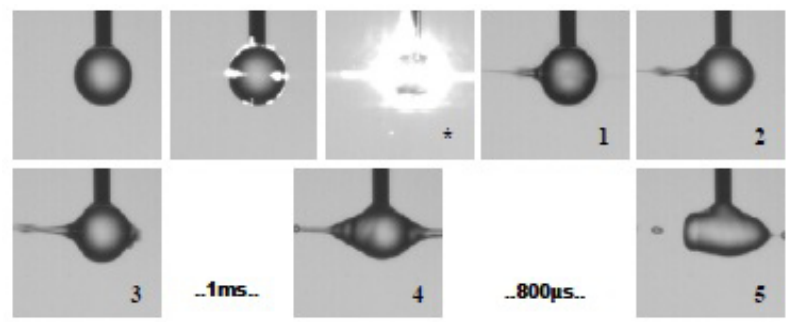

FIG. 11 Recording of the effects produced on the droplet by the laser beam sent in the equatorial plane and focused on the front face of the droplet. Droplet volume $3 \mu \mathrm{l}$; $\mathrm{E}_{4}=1 \mathrm{~mJ}$ Movie-6 (4,9 MB).

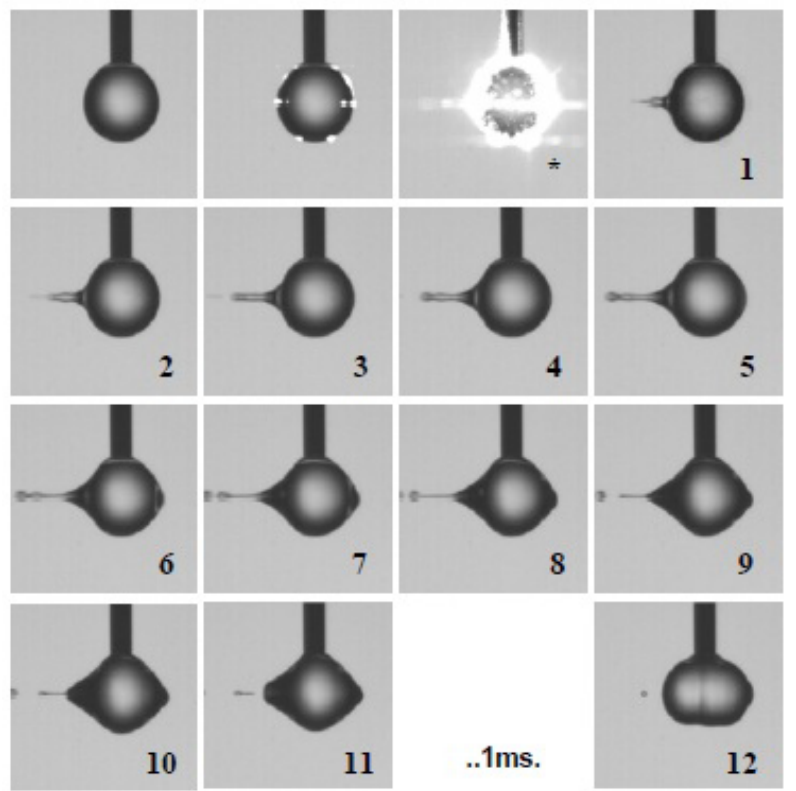

FIG. 12 Recording of the effects produced on the droplet by the laser beam sent in the droplet's equatorial plane and focused on its front face. Droplet volume $3.5 \mu \mathrm{l} ; \mathrm{E}_{1}=$ $0.25 \mathrm{~mJ}$ Movie-7 (4,9 MB). 
accompanied by modifications of the intensity distribution in the cross section of the beam.

When the laser beam has $1 \mathrm{~mJ}$, after more than $1 \mathrm{~ms}$ the front jet emits microdroplets in backward sense.

In all studied cases in this section the central jet is accompanied by a conical crown of small diameter droplets that has a more or less regulated form, function of the beam power, focus position and droplet volume/diameter. According to [6], such a formation is produced as a function of the deepness of the actual position of the focus inside the droplet which should be of the order of some tens of micrometers. This could be the case in our experiments although the conditions of experiments in [6] are largely different from our experimental conditions. The formation of such a crown could be due to the light pressure exerted by the more divergent rays in the focus (distributed at the periphery of the focus spot) on water.

This kind of effects may be understood considering that the water has a constant viscosity [28]; one should take into account the relation between the pressure applied by the laser beam on the droplet and the water viscosity within the droplet as well as the surface tension of it. At the interaction of the laser beam with the water droplet, a shear stress is produced so that the liquid tends to flow due to the light pressure which is applied by the beam on the droplet at the spherical interface with the air. Different layers of the water in the droplet move with various speeds which depend on the water viscosity. The shear stress $F$, may be computed if one considers the model described in [29]: the water is an homogeneous and isotropic liquid in which two plane plates are introduced at a the distance $y$ between them and having the surface area in the water $A$ larger than the area of the beam impact on the droplet; the plate/droplet boundary conditions may be neglected and a shear stress $F$ applied on a moving plate causes the water between the plates to undergo a shear flow with the velocity gradient $u$; if $\mu$ is the water viscosity, the shear stress is given by:

$$
F=\mu \cdot A \cdot u / y
$$

For the water, at $20^{\circ} \mathrm{C}, \mu=1.002 \mathrm{mPa} \cdot \mathrm{s}$ or $1.002 \cdot 10^{-3} \mathrm{Ns} / \mathrm{m}^{2}$.

On the other hand, $\mathrm{F}$ is in this case the pressure force $F_{p}$ which is given by

$$
F_{p}=E /(c \Delta t)
$$

where $E$ is the beam energy, $c$ is the light speed in water $(225,407,863 \mathrm{~m} / \mathrm{s})$ and $\Delta t=5 \mathrm{~ns}$ (laser pulse FTWHM) [30]).

Considering that the upper, fixed plate is the capillary that remains tangent at the droplet, in Table 3 the values of $u$ are given for beam energies and droplet diameters used in this work, produced by the laser beam focused on the front face of the droplet in the equatorial plane of it. One considers that the moving surface of area $A$ is the surface described by the laser beam in the droplet and the area $A$ is given by the product between the focus diameter on the droplet surface, $90 \mu \mathrm{m}$, and the respective droplet diameter. The computed $u$ values show that the speed of the water layers moved in the droplet by the laser beam has supersonic gradients which cover values

\begin{tabular}{|c|c|c|c|}
\hline \multirow{2}{*}{$\begin{array}{c}E \times 10^{-3} \\
(\mathrm{~J})\end{array}$} & \multirow{2}{*}{$\begin{array}{c}\mathrm{F} \times 10^{-3} \\
(\mathrm{~N})\end{array}$} & Diameter $\times 10^{-3}(\mathrm{~m})$ & \multirow{2}{*}{$\begin{array}{c}\mathrm{U} \\
(\mathrm{m} / \mathrm{s})\end{array}$} \\
\hline & & $\mathrm{y} \times 10^{-3}(\mathrm{~m})$ & \\
\hline \multirow{6}{*}{$E_{1}=0.25$} & \multirow[t]{6}{*}{0.22} & $\mathrm{D}_{1}=1.79$ & \multirow{2}{*}{$\begin{array}{c}u_{1}^{(1)} \\
=1219.8\end{array}$} \\
\hline & & 0.895 & \\
\hline & & $\mathrm{D}_{2}=1.88$ & \multirow{2}{*}{$\begin{array}{c}u_{1}^{(2)} \\
=1219.8\end{array}$} \\
\hline & & 0.94 & \\
\hline & & $\mathrm{D}_{3}=1.97$ & \multirow{2}{*}{$\begin{array}{c}u_{1}^{(3)} \\
=1219.8\end{array}$} \\
\hline & & 0.985 & \\
\hline \multirow[t]{6}{*}{$E_{2}=0.40$} & \multirow[t]{6}{*}{0.355} & $\mathrm{D}_{1}=1.79$ & \multirow{2}{*}{$\begin{array}{c}u_{2}^{(1)} \\
=1968.3\end{array}$} \\
\hline & & 0.895 & \\
\hline & & $\mathrm{D}_{2}=1.88$ & \multirow{2}{*}{$\begin{array}{c}u_{2}^{(2)} \\
=1968.3\end{array}$} \\
\hline & & 0.94 & \\
\hline & & $\mathrm{D}_{3}=1.97$ & \multirow{2}{*}{$\begin{array}{c}u_{2}^{(3)} \\
=1968.3\end{array}$} \\
\hline & & 0.985 & \\
\hline \multirow[t]{6}{*}{$E_{3}=\mathbf{0 . 7}$} & \multirow[t]{6}{*}{0.621} & $\mathrm{D}_{1}=1.79$ & \multirow{2}{*}{$\begin{array}{c}\mathrm{u}_{3}^{(1)} \\
=3443.1\end{array}$} \\
\hline & & 0.895 & \\
\hline & & $\mathrm{D}_{2}=1.88$ & \multirow{2}{*}{$\begin{aligned} & \mathrm{u}_{3}^{(2)} \\
= & 3443.1\end{aligned}$} \\
\hline & & 0.94 & \\
\hline & & $\mathrm{D}_{3}=1.97$ & \multirow{2}{*}{$\begin{aligned} & u_{3}^{(3)} \\
= & 3443.1\end{aligned}$} \\
\hline & & 0.985 & \\
\hline \multirow[t]{6}{*}{$\mathrm{E}_{4}=1$} & \multirow[t]{6}{*}{0.887} & $\mathrm{D}_{1}=1.79$ & \multirow{2}{*}{$\begin{aligned} & \mathrm{u}_{4}^{(1)} \\
= & 4917.9\end{aligned}$} \\
\hline & & 0.895 & \\
\hline & & $\mathrm{D}_{2}=1.88$ & $u_{4}^{(2)}$ \\
\hline & & 0.94 & $=4917.9$ \\
\hline & & $\mathrm{D}_{3}=1.97$ & $\mathrm{u}_{4}^{(3)}$ \\
\hline & & 0.985 & $=4917.9$ \\
\hline
\end{tabular}
from about $1200 \mathrm{~m} / \mathrm{s}$ to $4900 \mathrm{~m} / \mathrm{s}$ function of the beam en-
TABLE 3 Velocity gradients produced in the droplets by the laser beam at $532 \mathrm{~nm}$.

ergy and of the droplet's volume. From Table 3 it results that, because the capillary is hydrophobic, for a droplet volume the velocity gradient depends only on the pressure force/beam energy. The obtained values may justify the supersonic emission speeds of the jets or nanodroplets in the first phase of the interaction of the laser beams with the droplets.

As for the interaction speed of the laser beam with the water in the droplets, considering the speed of light in water (refractive index $n=1.33$ ) at room temperature and at normal air pressure, $c=225,407,863 \mathrm{~m} / \mathrm{s}$, the time intervals necessary for the light to cross the droplets are: for the $3 \mu \mathrm{l}\left(D_{1}=1.79 \mathrm{~mm}\right) t_{1}=7.94 \mathrm{ps}$, for the $3.5 \mu \mathrm{l}\left(D_{2}=1.88 \mathrm{~mm}\right) t_{2}=8.34 \mathrm{ps}$ and for the $4 \mu \mathrm{l}$ $\left(D_{3}=1.97 \mathrm{~mm}\right) t_{3}=8.74$ ps. In 8 ps the length of the propagation of the wave due to the velocity gradient varies from $9.75 \mathrm{~nm}$ $\left(E_{1}=0.25 \mathrm{~mJ}\right)$ to $39.3 \mathrm{~nm}\left(E_{4}=1 \mathrm{~mJ}\right)$ so that at the recording speeds used, one may consider that during the propagation of the laser beam through the droplet, this one remains unchanged.

All the produced processes take place at lower speed and are measurable after the laser beam interaction with the droplet's material ended. More, considering that the laser pulse lifetime in the droplet is $5 \mathrm{~ns}$, after the first photons leave the droplet, there are still incoming photons for a time interval around 4.992 ns. Given the velocity gradients computed in Table 3 the time necessary for the wave produced by the laser beam in the 
droplet to propagate in it, is around $1.5 \mu \mathrm{s}$ for $E_{1}=0.25 \mathrm{~mJ}$ and $0.4 \mu \mathrm{s}$ for $E_{4}=1 \mathrm{~mJ}$ and there is no time for the laser beam to interact directly within the droplet with the pressure waves produced by it.

Another process which is evidenced in all the figures of this chapter is the production of small droplets along the laser beam propagation direction in the backward and forward sense. This may be observed in all pictures.

According to $[6,25,26,31]$ the laser beam may induce electrostriction in the water droplet which leads to the formation of a bulge.

In this paper more bulges may be observed in some cases and an individual bulge has a conical shape that evolves so that at the tip, a cylinder is formed having the diameter of the cross section of some micrometers. The surface tension pulls the cylinder which is detached from the cone and forms a droplet that has some micrometers diameter whereas the remaining long cone with the basis in the droplet evolves towards being again integrated in it. This process may take from a few hundreds of $\mu$ s to some ms. In this paper this may be seen in Figures 10 (frames 8 and 9), 12 (frames 5, 6, 7), 13 (frames 4 and 5), 18 (frames 9 and 10) and 19 (frames 2 and 3).

According to [25] the surface tension forces applied at the tip of the cone generated from the main droplet is

$$
F=2 \pi \cdot r \cdot \sigma \cdot \sin \theta
$$

where $r$ is the radius of the cone section measured from the axis of the cylinder, $\sigma$ is the water surface tension and $\theta$ is the angle that is made by the normal at the cone surface with the mentioned axis.

In other words one may consider that $F$ is the threshold starting from which the droplets may be detached from the cone. If $F$ is known, one may compute $r$ considering that this radius is that of the produced droplet, $\sigma=(72.4 \pm 0.6) \times 10^{-3} \mathrm{~N} / \mathrm{m}$ extracted from [1] and $\sin \theta$ around 1 . The forces which contribute to the formation of the cone are the pressure force exerted by the laser beam on the droplet and the shear stress force due to the viscosity of the water that has the values computed in Table 3 and may be opposed to the light pressure force since the water is isotropic.

The light pressure force is computed as shown above; $c$ is the speed of light in air, i.e. 299,102,690 m/s [30]. For the beam energies used, the laser beam pressure forces are: for $E_{1}=0.25 \mathrm{~mJ}$, $0.166 \times 10^{-3} \mathrm{~N}$; for $E_{2}=0.40 \mathrm{~mJ}, 0.266 \times 10^{-3} \mathrm{~N}$; for $E_{3}=0.70 \mathrm{~mJ}$, $0.466 \times 10^{-3} \mathrm{~N}$; and for $E_{4}=1.0 \mathrm{~mJ}, 0.666 \times 10^{-3} \mathrm{~N}$.

Using these values and those given in Table 3, the corresponding $r$ values are: for $E_{1}, 0.118 \mathrm{~mm}$; for $E_{2}, 0.196 \mathrm{~mm}$; for $E_{3}$, $0.34 \mathrm{~mm}$ and for $E_{4}, 0.486 \mathrm{~mm}$. The measured diameters of the microdroplets expulsed in the pictures in this section are found in this dimensions range.

The computation based on the images shown in figures is approximate. The optical arrangement used to record images does not allow the accurate measurement of the propagation

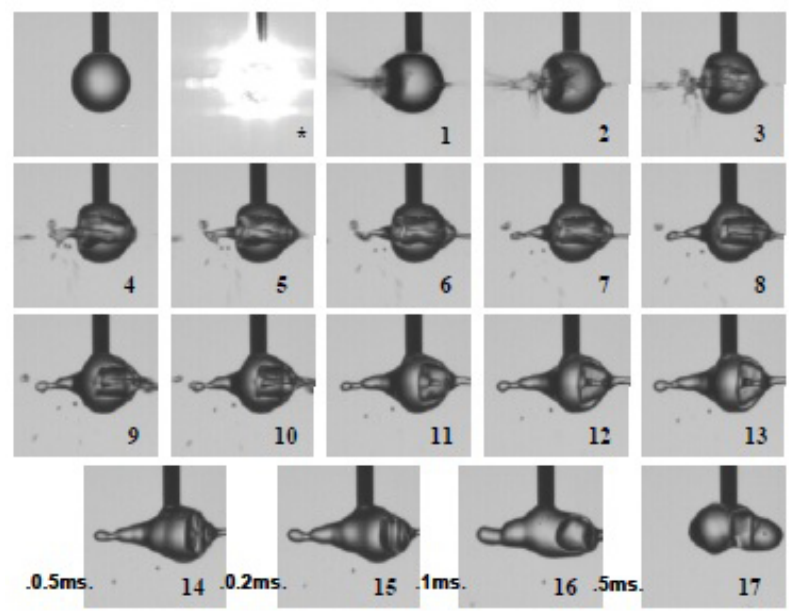

FIG. 13 Recording of the effects produced on the droplet by the laser beam sent in the droplet's equatorial plane and focused on its front face. Droplet volume $3.5 \mu \mathrm{l} ; \mathrm{E}_{2}=$ $0.4 \mathrm{~mJ}$ Movie-8 (4,9 MB).

length of the nanodroplets immediately after the impact of the laser beam with the droplet. This is mainly due to the high intensity of the light scattered by the droplet and possibly to the triggering of the high speed camera. To improve the contrast, in Figure 16 are shown images obtained by introducing a partially reflecting mirror between the droplet and the camera that cuts-off $532 \mathrm{~nm}$ light. The pictures have 256x256 pixels resolution and for preserving the total number of pixels, if the picture's length is increased, its width is diminished accordingly.

The frame $\left(^{*}\right)$ in Figure 16 shows that the nanodroplets emitted after the interaction with the laser beam propagate with a supersonic speed of about $450 \mathrm{~m} / \mathrm{s}$, faster than in Figures 9 frame $\left(^{*}\right)$ or 11 frame $(*)$. This result is closer to reports showing expulsion of droplets at speeds around $1000 \mathrm{~m} / \mathrm{s}$ after interaction of water droplets with pulsed laser beams (FTW $17 \mathrm{~ns}$ ) emitted at $248 \mathrm{~nm}$ and at $3 \mathrm{GW} / \mathrm{cm}^{2}$ power density [21]. The same kind of results are reported in [22] where a Nd:YAG pulsed laser (FTW 10ns) is used that emits at $1.064 \mu \mathrm{m}$ a beam of $20 \mathrm{~J} / \mathrm{cm}^{2}$ fluence. In Figure 16, the power density of the laser beam was $3.44 \mathrm{GW} / \mathrm{cm}^{2}$ (Table 2); it is possible that the visualised jet may be shorter than in reality and this is why the calculated speed is lower than $1000 \mathrm{~m} / \mathrm{s}$.

In the cases in which the focus of the laser beam is positioned accurately on the droplet surface, the distribution of intensity in the cross section of the focal plane may produce effects on the droplet like in Figure 18 frames 3 to 9. The two liquid cylinders coming out from the droplet (Figure 18) in the forward sense may be due to the fact that, the laser beam although being single mode does not have a Gaussian intensity distribution in the cross section, particularly in the focus. There are two intensity peaks on the vertical which propagate through the droplet and produce two divergent jets as a consequence of the energy densities of the light beam within the droplet. For understanding this effect one should also consider that the laser beam has a waist of $90 \mu \mathrm{m}$ that is preceded by larger areas (see pictures before frame B in Figure 3) more than $1 \mathrm{~mm}$ and the intensity distribution in the corresponding cross sections are showing two peaks. This effect may be correlated 


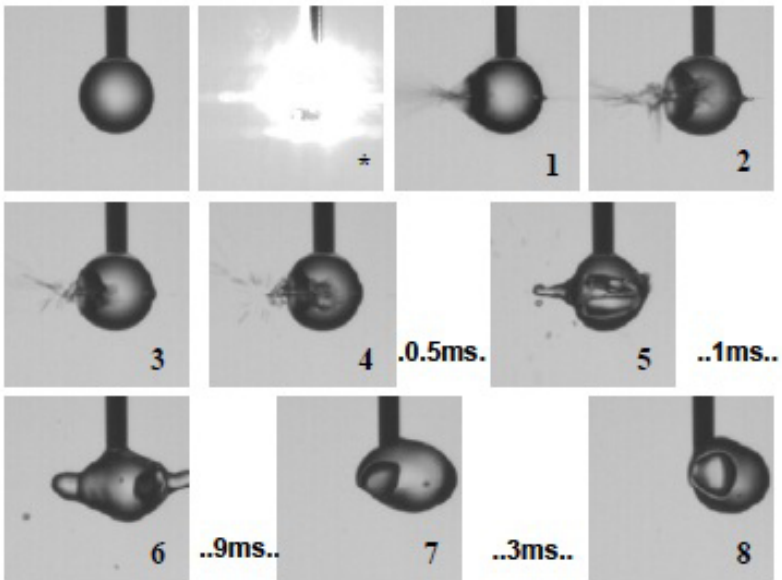

FIG. 14 Recording of the effects produced on the droplet by the laser beam sent in the droplet's equatorial plane and focused on its front face. Droplet volume $3.5 \mu \mathrm{l} ; \mathrm{E}_{3}=$ $0.7 \mathrm{~mJ}$ Movie-9 (4,9 MB).

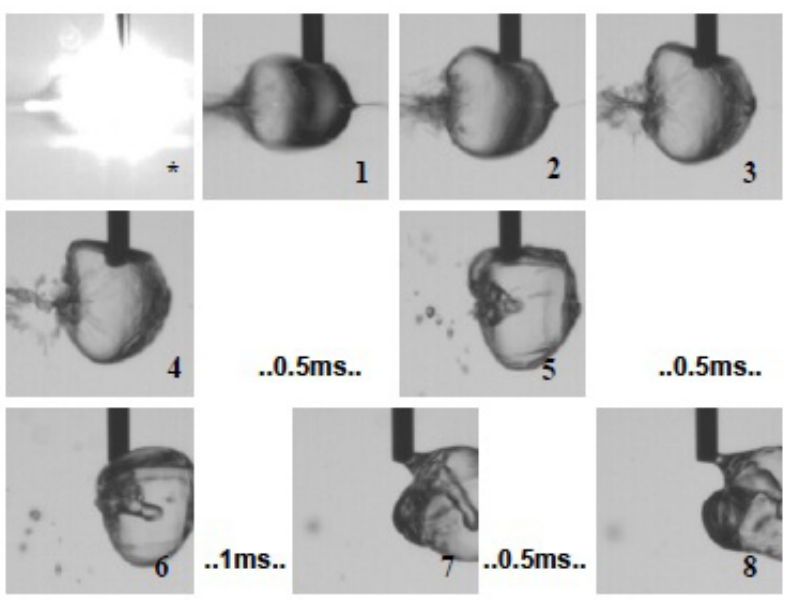

FIG. 15 Recording of the effects produced on the droplet by the laser beam sent in the equatorial droplet's plane and focused on its front face. Droplet volume $3.5 \mu \mathrm{l} ; \mathrm{E}_{4}=$ $1 \mathrm{~mJ}$ Movie-10 (4,9 MB).

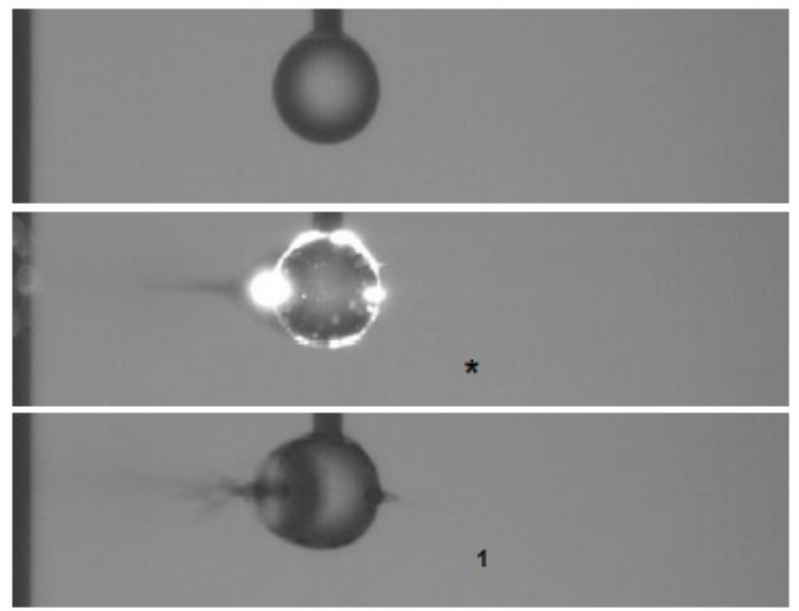

FIG. 16 Recording of the effects produced on the droplet by the laser beam sent as in Figure 14. Droplet volume $3.5 \mu \mathrm{l} ; \mathrm{E}_{4}=1.0 \mathrm{~mJ}$. Partially reflecting mirror at $532 \mathrm{~nm}$ introduced between the droplet and the high speed camera Movie-11 (4,9 MB).

with results reported in [22] where measurements of the pulse laser ablation on a liquid surface were made in a different geometry than the pendant droplet hanging in air and in

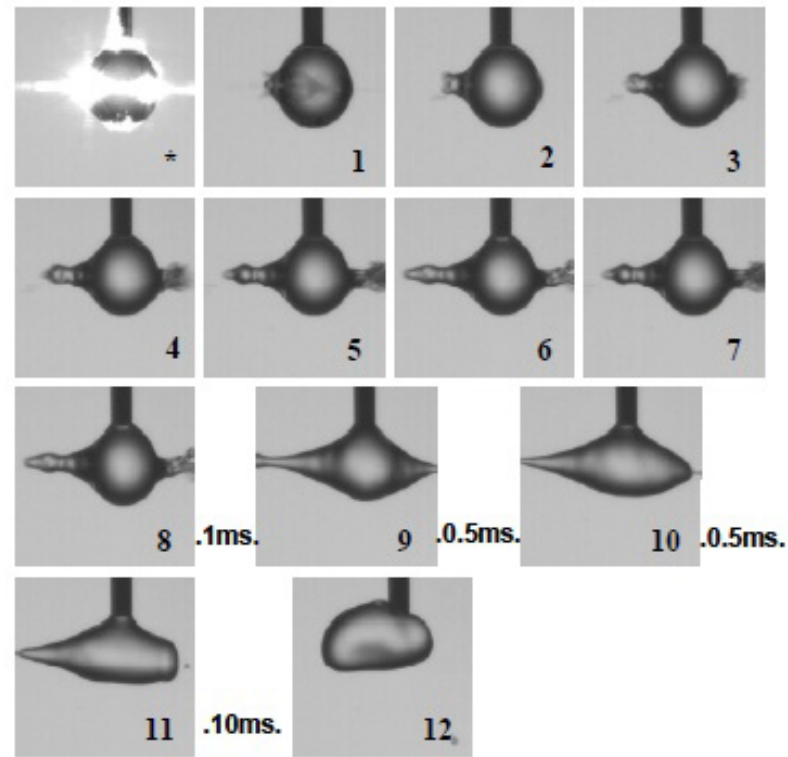

FIG. 17 Recording of the effects produced on the droplet by the laser beam sent in its equatorial plane and focused on its front face. Droplet volume $4 \mu \mathrm{l} ; \mathrm{E}_{1}=0.25 \mathrm{~mJ}$ Movie-12 (4,9 MB).

other focusing conditions. In [22] it was shown that the shape and behaviour of the expulsed material from the water surface at the interaction with a focused laser beam sent from water onto air depend on the intensity distribution within the cross section of the beam: top-hat, Gaussian and torus distributions were checked. Typical examples of this kind may be seen in Figures 9- 17 and 19 where the laser beam is focused on the front surface of the droplet, perpendicular to the surface. The deformations have conical forms that may propagate backward or forward with respect to the laser beam propagation; they are function of the intensity distribution in the focus of the beam and of the accuracy with which the focus is positioned on the droplet's surface. If there are more intensity peaks in the cross section of the focus, it may be possible to obtain more conical deformations as in Figure 18. According to the figures shown in this section, the deformation cones are developed in at least three stages:

- the first, very fast, during which micro and nanodroplets are expulsed at supersonic speeds, or even a very thin liquid cylinder is produced (typically Figure 16);

- the second that is slower; during it a larger cone with the basis of hundreds of $\mu \mathrm{m}$ is formed. Towards to end of this stage one or more larger droplets are expulsed from the tip of the cone, at much lower speeds, as may be observed in Figures 9 frame 13, 10 frame 8,11 frames 4 and 5, 12 frames $5-12$. The dimensions of the expelled droplets vary from one case to another. The propagation speed of these microdroplets are of the order of some tens of $\mathrm{m} / \mathrm{s}$;

- the third, is the retraction of the cone in the water droplet which is actually a damping of the oscillations produced by the interaction with the laser beam and which may take milliseconds to reach the equilibrium state.

The impact of the laser beam may have also violent/explosive 


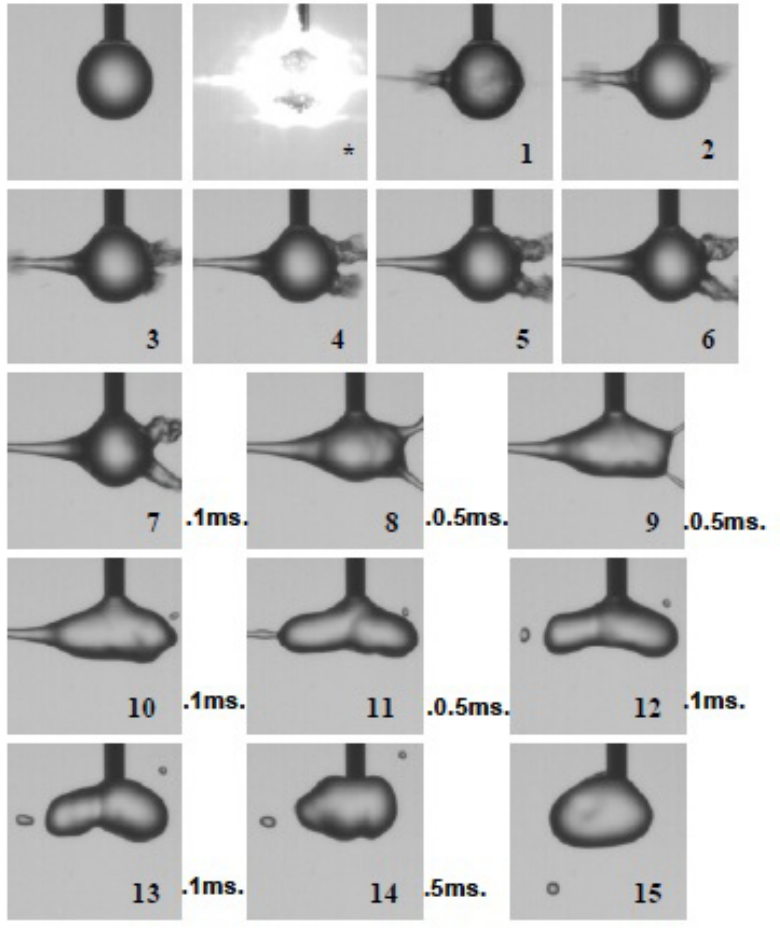

FIG. 18 Recording of the effects produced on the droplet by the laser beam sent in the droplet's equatorial plane and focused on its front face. Droplet volume $4 \mu \mathrm{l} ; \mathrm{E}_{2}=$ $0.4 \mathrm{~mJ}$ Movie-13 (4,9 MB).

effects which are described by some authors as ablation processes. First, one should observe that even for nonviolent effects, the formation of the backward central cones starting from the focus placed on the front point of the surface, accompanied by the conic collars is supplied from the surrounding water, so that in the droplet is formed an air cylinder that is coaxial with the cone and that propagates the more within the droplets the longer the cone (Figure 13 frames 3 and 4, Figure 14 frame 5).

After the laser beam is entering in the second half of the droplet and passing through its centre, the light pressure at the output surface produces a forward cone which also extracts water from its neighbourhood in the droplet. So, function of the fluence of the laser beam, the focus position and the droplet volume, it is possible that the cylinder breaks through the droplet for a short time or, at least, a large air bubble and possibly smaller diameter air bubbles are formed in the droplet. This effect may be seen in Figures 10, 13, 14, 19. If the pulse energy is $1 \mathrm{~mJ}$, one obtains explosive effects on the droplet which eventually destroy it as in Figure 15. This process takes place in a few milliseconds and is produced by shaping air bubbles in the water droplet and by detaching the droplet from the capillary on which it is hanged.

\section{1 .3}

Interaction of the laser beam incident on the droplet in the equatorial plane and focused on its back face (position 3 in Figure 2).

If one places the focus in the equatorial plane on the back sur-

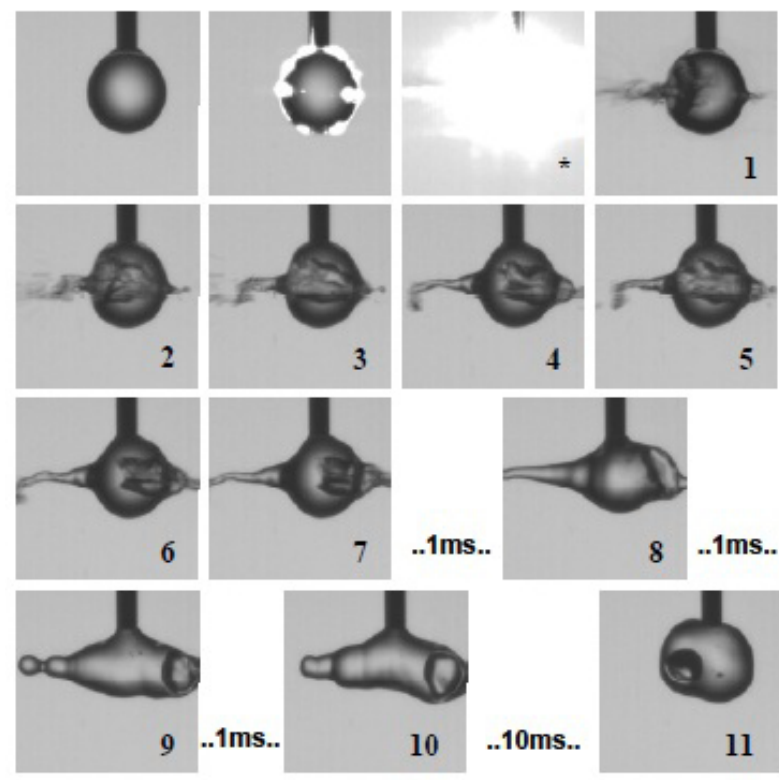

FIG. 19 Recording of the effects produced on the droplet by the laser beam sent in droplet's equatorial plane and focused on its front face. Droplet volume $4 \mu \mathrm{l} ; \mathrm{E}_{3}=$ $0.7 \mathrm{~mJ}$ Movie-14 (4,9 MB).

face, so that the beam is perpendicular to it one obtains the same kind of effects as if the beam is focused on the front.

In Figure 20 the unresonant interaction effects of a $0.4 \mathrm{~mJ}$ beam on a $3 \mu \mathrm{l}$ droplet are shown. The front surface effects have a lower intensity and the processes on the back, show the formation of the central cone surrounded by the droplets collar. The cone and the collar propagate with the speeds calculated in Section 3.1.2 and the process ends with the generation of small size (tens of $\mu \mathrm{m}$ ) droplets and the detachment of the droplet from the capillary.

The shapes of the deformations produced by the pressure of the laser beam as shown in Figure 20 frames 9-10, or Figure 21 frames 3 to 10, have the same forms as described in [12] for fluid - fluid interface deformation by exposure of one fluid to a cw laser wave. In our experiments such effects were obtained for a pulsed laser beam that is weakly absorbed by water and that has fluences between 4 and $17 \mathrm{~J} / \mathrm{cm}^{2}$ (Table 2) when it is focused at different positions on the surface of a hanging droplet in air.

When the focus is centred in the symmetry centre of the droplet such effects are not obtained or are even covered by the processes triggered by the electrostrictive unhomogeneties produced inside the droplet.

In Figure 21 the detachment of the droplet from the capillary is produced after $9.1 \mathrm{~ms}$ after the interaction with the laser beam of $0.70 \mathrm{~mJ}$ energy, which is shorter than the corresponding time in Figure $20(10.6 \mathrm{~ms})$ for a droplet with the same volume $(3 \mu \mathrm{l})$ but exposed to $0.4 \mathrm{~mJ}$ beam.

If the energy is increased, besides the cone structure formation, one obtains an effect similar to the electrostrictive effect reported in Section 3.1.1 by focusing the beam in the centre of the droplet. The magnitude of it is not as high as in Sec- 


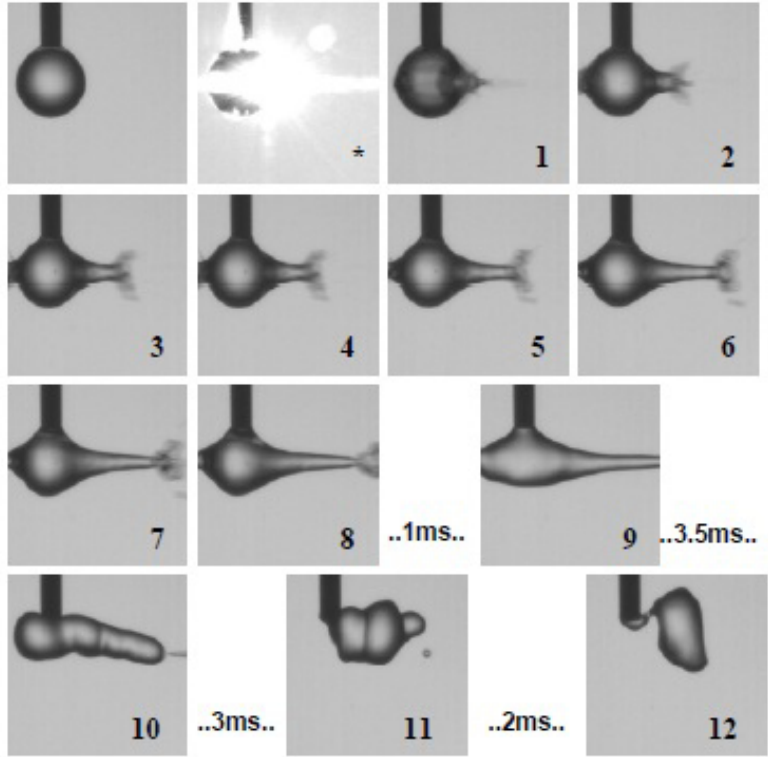

FIC. 20 Recording of the effects produced on the droplet by the laser beam sent in its equatorial plane and focused on the back face of it. Droplet volume $3 \mu \mathrm{l} ; \mathrm{E}_{2}=0.4 \mathrm{~m}$ J Movie-15 (4,9 MB).

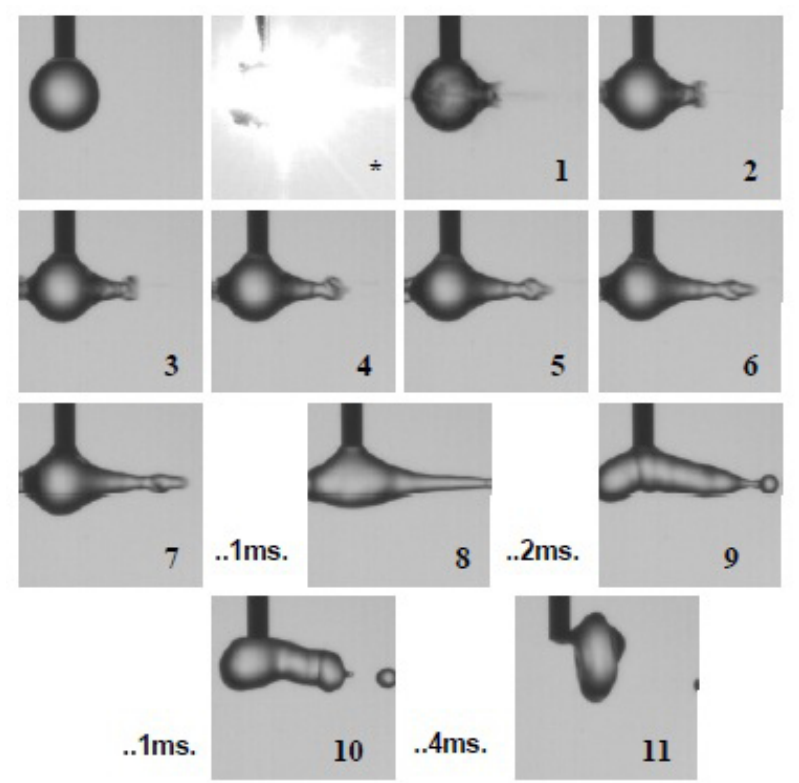

FIG. 21 Recording of the effects produced on the droplet by the laser beam sent in the equatorial plane and focused on the back face of it. Droplet volume $3 \mu \mathrm{l} ; \mathrm{E}_{3}=0.70 \mathrm{~mJ}$ Movie-16 (4,9 MB).

tion 3.1.1 because the beam power density in the droplet is not as high as in Figures 7 and 8, but significant expulsions of water jets and droplets take place on directions other than backward, forward and the associated collars, as results from Figure 22 where laser beam has $1 \mathrm{~mJ}$ energy. This may be correlated with the laser beam waist before the focus, in the focus and after it, as results from Figure 3 where the distance on the OX direction between two successive images of the beam cross section is $250 \mu \mathrm{m}$. So, for the exposure position shown in Figure 22, if the focus shown in picture A (Figure 3) is placed on the back surface, the area of the cross section of the laser beam at the input in the droplet is around $65,600 \mu \mathrm{m}^{2}$ that is about 10.3 times larger than the area of the focus in picture A

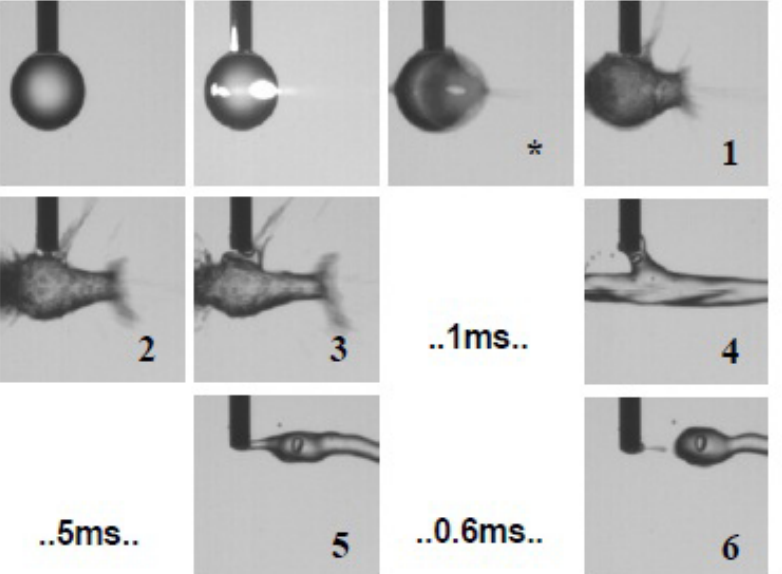

FIG. 22 Recording of the effects produced on the droplet by the laser beam sent in its equatorial plane and focused on its back face. Droplet volume $3.5 \mu \mathrm{l} ; \mathrm{E}_{4}=1 \mathrm{~mJ}$ Movie-17 (4,9 MB).

$\left(6,358 \mu \mathrm{m}^{2}\right)$. This means that the energy density of the beam in the focus is about 10.3 times higher than at the entrance of the beam in the droplet and the conditions to reach the threshold to produce light scattering by the electrostrictive inhomogeneities are not met.

In Figure 22 the frame ${ }^{*}$ ) shows the droplet's shape at the start of the conic structure formation which indicates a strong deformation of the second hemisphere of the droplet followed by the cone and collar formation and movement. The characteristics of the emitted jets in forward direction are somewhat similar with those described in 3.1.2. On the other hand, in Figure 22, the respective droplets are detached from the capillary, the detachment times being around $7 \mathrm{~ms}$; they are shorter than in Figure 21 and this may be due to the higher energy of the pumping laser beams in Figure 22 combined with the larger volumes. The observation related to faster detachment times for larger/heavier droplets could be an argument in the favour of the hypothesis that the water behaves in these experiments like a Newtonian fluid with specific inertial properties.

A final observation about the interaction in this arrangement is that the capillary which is touched by the waves produced within the droplet contribute to the expulsion of jets of liquid or of droplets of micrometric dimensions, as results from Figure 22.

\section{1 .4}

Interaction of the laser beam incident on the droplet in the equatorial plane, on the lateral face of it, located in front of the camera (position 8 in Figure 2).

The results reported in this section may be considered as similar with those presented in Section 3.1.2 because in both cases the laser beam comes from the environment (air) and interacts with the water droplet from outside towards the inside of it. The main difference is that position 8 on the droplet's surface, in Figure 2, is placed just in front of the high speed camera so that the images show the effects obtained on the water droplet 
after the unresonant interaction, seen along the radius of the droplet.

The waist of the laser beam has $90 \mu \mathrm{m}$ diameter and in order to obtain effects on the droplet, the focal spot must be positioned so that it is partially or totally included within the droplet in the immediate proximity of the surface. At this position, the laser beam is refracted by the droplet's material so that a part of it is further sent outside the droplet as described in [32], i.e. in a sense opposite to the high speed camera. This allows the obtaining of pictures with a better contrast since the intensity of the parasite light background is lower.

The pressure effects will take place mainly along the radius of the droplet in the contact point, although the beam is practically tangent to the droplet surface. The effects are of the same kind as in Section 3.1.2, i.e. they are developed as three step processes. In Figure 23 the dynamics of the process is shown. One may observe the initial deformation and water cone/cylinder produced by the laser beam (energy: $0.4 \mathrm{~mJ}$, beam power $80 \mathrm{~kW}$, fluence $6.89 \mathrm{~J} / \mathrm{cm}^{2}$, beam power density $1.38 \mathrm{GW} / \mathrm{cm}^{2}$ ) at the droplet's surface, in the first tens of $\mu \mathrm{s}$. The droplet's deformations that are perpendicular on the droplet's surface and high speed camera observation plane are better evidenced in frame 3 (Figure 23) at about $200 \mu \mathrm{s}$ from the impact with the laser beam. After $200 \mu \mathrm{s}$, the back surface emission is noticed and the formation of the liquid cone and the associated cylinder hole in the droplet, accompanied by emissions of micro- and, possibly, nano-droplets takes place. The droplets are recorded as a smoke like image (frames 4 16) and as it results from frames 8 and 9 -plume, labelled $\left({ }^{\circ}\right)$ - their propagation speed is in the range $2-3 \mathrm{~m} / \mathrm{s}$.

The impact with the beam produces a slight rotation of the droplet that is related with its elongation and the decrease of its transverse diameter until the remaining droplet is detached from the capillary after around $4.8 \mathrm{~ms}$.

In Figure 24 are shown the same kind of effects produced on a droplet of $3.5 \mu \mathrm{l}$ volume, when the laser beam has a higher energy, $0.7 \mathrm{~mJ}$ (power $140 \mathrm{~kW}$, fluence $12.06 \mathrm{~J} / \mathrm{cm}^{2}$, power density $\left.2.41 \mathrm{GW} / \mathrm{cm}^{2}\right)$. One observes the central cone and the surrounding air cylinder; the micro- and nano-droplets around the cone look like a smoke, but larger droplets are also generated such as shown in frames 10 or 16. These relatively larger microdroplets propagate during a $100 \mu$ s record so that they appear sometimes like a line with a given length such as in frames 3 and 4 where the moving droplet labelled $\left({ }^{\circ}\right)$ has a computed speed of about $4 \mathrm{~m} / \mathrm{s}$. The dimension of the pendant droplet decreases as well, once the water cone and the associated cylinder are built so that after $32.2 \mathrm{~ms}$ the droplet is detached from the capillary. The detached material may be organised in some larger droplets that may contain air bubbles (Figure 24, frame 17).

\section{1 .5}

Laser beam interaction with the droplet, when sent horizontally, at the South Pole of it (position 4 in Figure 2).

When the laser beam is sent horizontally and focused on the

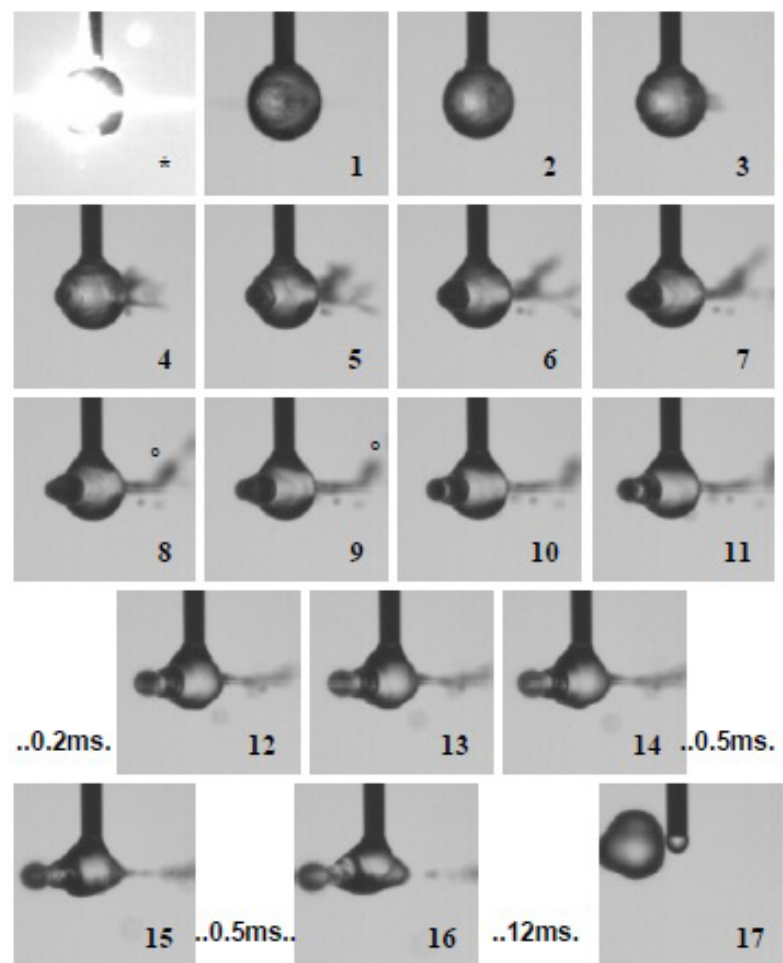

FIG. 23 Recording of the effects produced on the droplet by the laser beam focused on the lateral face of it, located in front of the camera. Droplet volume $3.5 \mu \mathrm{l} ; \mathrm{E}_{2}=0.4 \mathrm{~m}$ J Movie-18 (4,9 MB).

South Pole of the droplet one of the main parameters to control is the position of the focus on the surface, i.e. to control if all the $90 \mu \mathrm{m}$ diameter spot is included within the droplet under its surface or only a fraction of it. If the focus spot is placed in the droplet, refraction phenomena [32] and total reflection of the laser beam at the droplet interface with the air are produced, so that the light wave propagates under the surface within a thin layer [12]. Figure 25 shows what happens when the focus is not fully included in the droplet. The totally reflected light touches the capillary - see the frame $\left({ }^{*}\right)$ - and this leads to the fast (about $100 \mu \mathrm{s}$ ) emission of a conic jet near the contact area with the capillary, that may be finalised after some hundreds of microseconds with the emission of nanodroplets (frames 2 and 3 in Figure 25). The cone is perpendicular on the surface $[7,12]$. The processes are not explosive because the beam energy is low; the acoustic waves produced in the droplet by composing the light pressure with the water shear stress and with the surface tension lead to droplet's damped oscillations and deformations keeping it in pendant position.

If the beam energy is increased to $0.70 \mathrm{~mJ}$ and the focus position is kept as in Figure 25 one obtains the effects shown in Figure 26. The total reflection of light inside the droplet, close to its surface leads to a more intense circular layer of radiation - frame $\left(^{*}\right)$ in Figure 26; the interaction with the capillary leads to explosive emission of material in the neighbourhood of the capillary tip (frames $1-4$ ). Once entered inside the droplet, the first contact of the laser beam with the droplet surface produces a typical cone - collar formation which propagates with relatively high speed; from frames 1 and 2 in Figure 26 the cone labelled $\left({ }^{\circ}\right)$ elongates with more than $130 \mathrm{~m} / \mathrm{s}$. The cone 


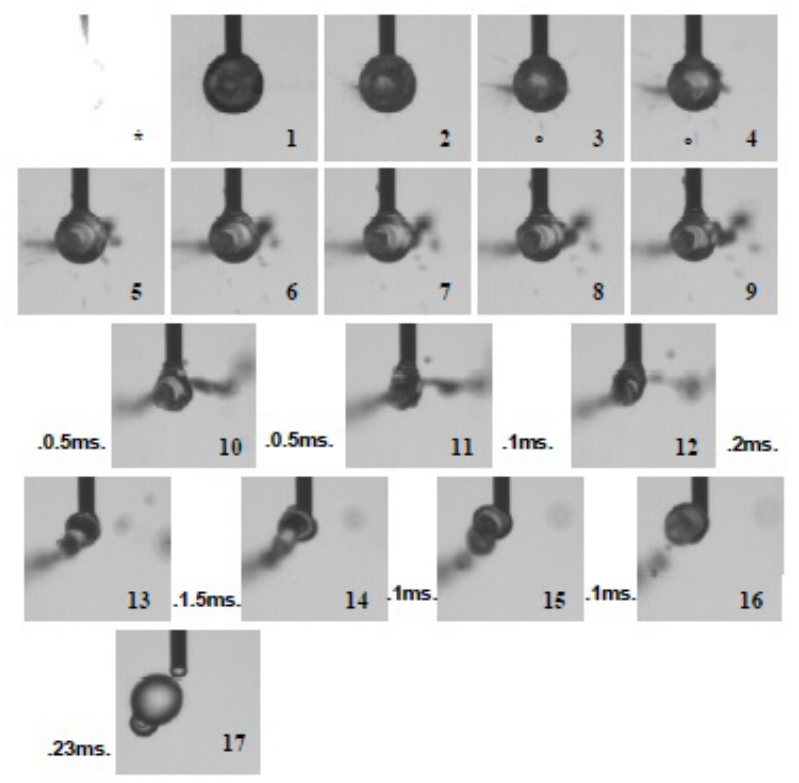

FIG. 24 Recording of the effects produced on the droplet by the laser beam sent in droplet's equatorial plane and focused on its face located in front of the camera. Droplet volume $3.5 \mu \mathrm{l} ; \mathrm{E}_{3}=0.7 \mathrm{~mJ}$ Movie-19 (4,9 MB).

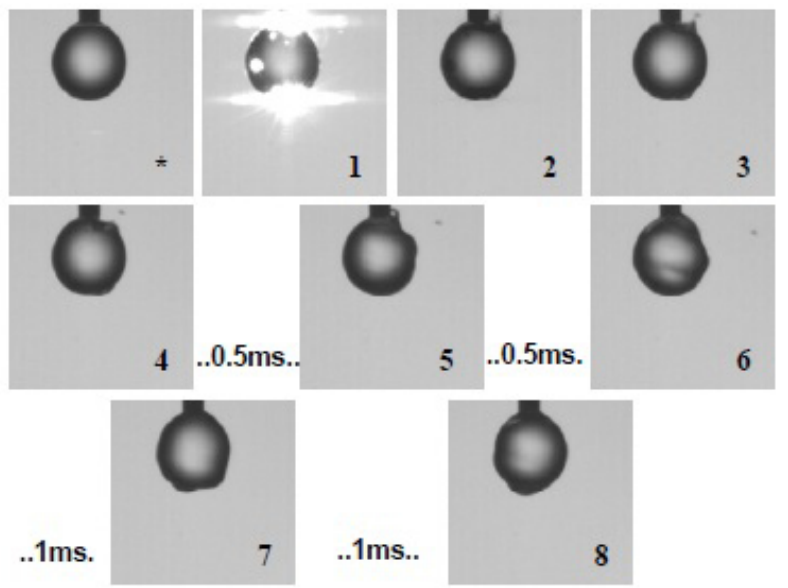

FIG. 25 Recording of the effects produced on the droplet by the laser beam sent horizontally and focused at the South Pole of it. Droplet volume $3.5 \mu \mathrm{l} ; \mathrm{E}_{1}=0.25 \mathrm{~m}$ J Movie-20 (4,9 MB).

generates finally micrometric droplets and the main droplet is detached from the capillary after about $2.1 \mathrm{~ms}$. The cone production is somewhat equivalent with the production of whipping fluid jets by electrospinning which generate submicron jets $[10,22]$ or polymer fibers $[32,33]$.

The characteristics of the jets like that shown in Figure 26 make them close to the Taylor steady coaxial jets of immiscible liquids, generated by electro-hydrodynamic forces [34].

The focused beam induced relatively important electrostrictive unhomogeneities that lead to light scattering in the droplet and the associated emission of water jets, according to the processes described in Section 3.1.1. In this case the capillary plays its role in enhancing the emission of jets in its neighbourhood and finally in the detachment of the droplet from it, in about $9.5 \mathrm{~ms}$.

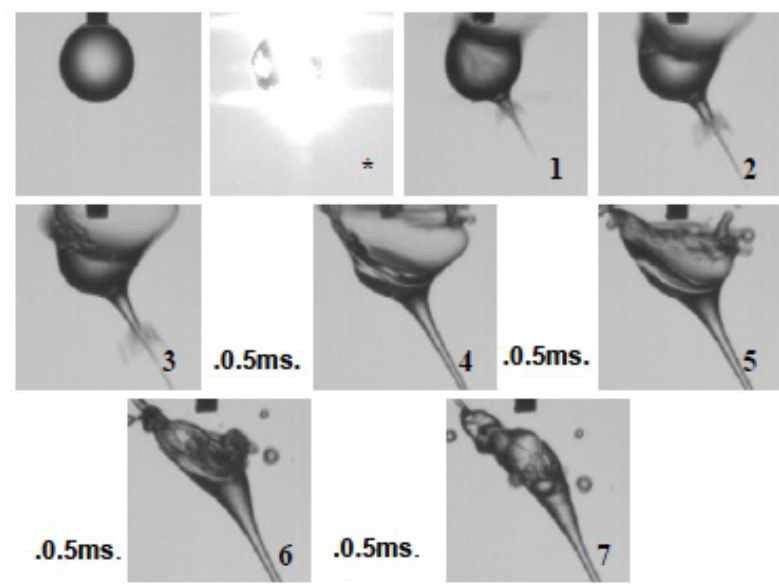

FIG. 26 Recording of the effects produced on the droplet by the laser beam sent horizontally and focused at the South Pole of it. Droplet volume $3.5 \mu \mathrm{l} ; \mathrm{E}_{3}=0.7 \mathrm{~mJ}$ Movie-21 (4,9 MB).

\subsection{Resonant interaction}

The resonant interaction studies were performed on droplets containing R6G solutions in distilled water at $10^{-3} \mathrm{M}$ concentration. R6G is one of the most utilised dyes for tuneable laser beam generation and it is well characterised from the optical, spectral and thermal points of view [20,35]. Its quantum efficiencies in absorbing and emitting radiation are high and make it one of the most efficient fluorophores in water or alcohol solutions. In this paper, the water is used as solvent because it has low evaporation rates and the droplets do not loose material during the experiments by evaporation.

The droplets used had $3 \mu \mathrm{l}$ volume (1.79 mm diameter) and the laser beam at $532 \mathrm{~nm}$ interacted with them only in positions 1, 2 and 3 (Figure 2). The R6G solutions in water strongly absorb in green, at $532 \mathrm{~nm}$.

A synthesis of the results is presented in Figures 27 to 32 which contain, each, a movie showing the dynamics of the droplet and relevant frames of the corresponding movie.

In Figure 27 the results of the laser beam interaction at $\mathrm{E}_{1}=$ $0.25 \mathrm{~mJ}$ with the droplet are shown, when the focus is placed in the centre of it (position 1 in Figure 2). The frame $\left(^{*}\right)$ shows an intense emission of fluorescence radiation after the absorption of the $532 \mathrm{~nm}$ beam. The next frames show that the droplet does not suffer any deformation, mechanical vibrations or loss of material. It seems that in frame 1 some unhomogeneity is produced, but no significant mechanical effects are observed. The reason is that the R6G at $10^{-3} \mathrm{M}$ in the water solution absorbs the beam at $532 \mathrm{~nm}$ so that the most photons of it produce the excitation of the R6G molecules on excited singlet states. The process takes place in the first layers of the droplets and there are not available photons to transfer their momentum to the droplet's water and to build significant light pressure effects on it.

If the beam remains focused in the centre of the droplet but its energy is increased at $1 \mathrm{~mJ}$ as may be seen in Figure 28, the interaction produces in the first $10 \mu \mathrm{s}$ - frame $\left(^{*}\right)$ - besides the strong emission of fluorescence radiation a fast loss of mate- 


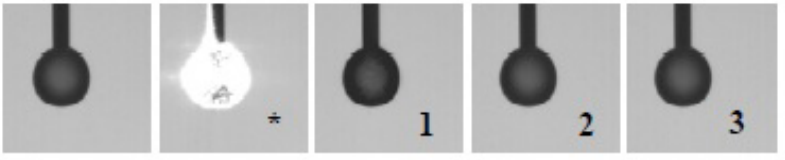

FIC. 27 Recording of the effects produced at resonant interaction on the droplet containing R6C at $10^{-3} \mathrm{M}$ in distilled water by the laser beam sent in its equatorial plane and focused in its centre (position 1 Figure 2). $E_{1}=0.25 \mathrm{~mJ}$ Movie-22 (4,9 MB).
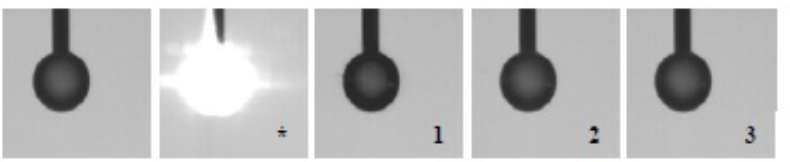

FIG. 28 Recording of the effects produced at resonant interaction on the droplet containing R6G at $10^{-3} \mathrm{M}$ in distilled water by the laser beam sent horizontally and focused in the centre of it (position1 Figure 2). $\mathrm{E}_{4}=1 \mathrm{~mJ}$ Movie-23 (4,9 MB).

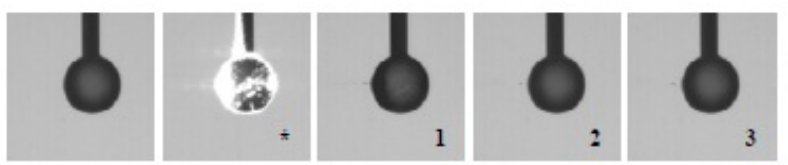

FIG. 29 Recording of the effects produced in resonant interaction conditions on the droplet containing R6G at $10^{-3} \mathrm{M}$ in distilled water by the laser beam focused on the front surface of it (position 2 in Figure 2). $E_{2}=0.4 \mathrm{~mJ}$ Movie-24 (4,9 MB).

rial. This effect has not a significant amplitude and the next frames (1 to 3 ) do not show important deformations or vibrations of the droplet.

Though, in frame 1 a weak backward structured emission may be observed at the input face which is either a tinny solution jet or a succession of micro and nanodroplets which propagate at lower speed. The strong absorption of the $532 \mathrm{~nm}$ radiation by the R6G molecules leaves available a relatively small number of photons which may apply pressure on the droplet. In understanding the interaction of the laser beam with the droplet, one should take into account, particularly in this case, the shape of the laser beam in the neighbourhood of the waist (Figure 3), which makes available relatively constant photon densities 250 - $500 \mu \mathrm{m}$ along the beam propagation direction.

When the focus is placed in position 2 (Figure 2) the effects on the droplet after resonant interaction with the $532 \mathrm{~nm}$ beam are shown in Figures 29-31.

In Figure 29, a $0.4 \mathrm{~mJ}$ beam energy is used and one obtains a strong fluorescence radiation followed by very small light pressure effects; in frame 1 a tinny backward jet is produced that is not accompanied further by significant deformation effects on the droplet. In frame $\left(^{*}\right)$ one observes fluorescence radiation emitted at the input and output of the laser beam on/from the droplet which may indicate either higher photon densities in those volumes or a density gradient in the same points as a consequence of the effects of the laser beam. The droplet's deformation remains very low, but one observes in frames 1, 2 and 3 the formation of the backward cone at the front surface that generates after some hundreds of $\mu \mathrm{s}$ one or more nanodroplets which propagate with relatively

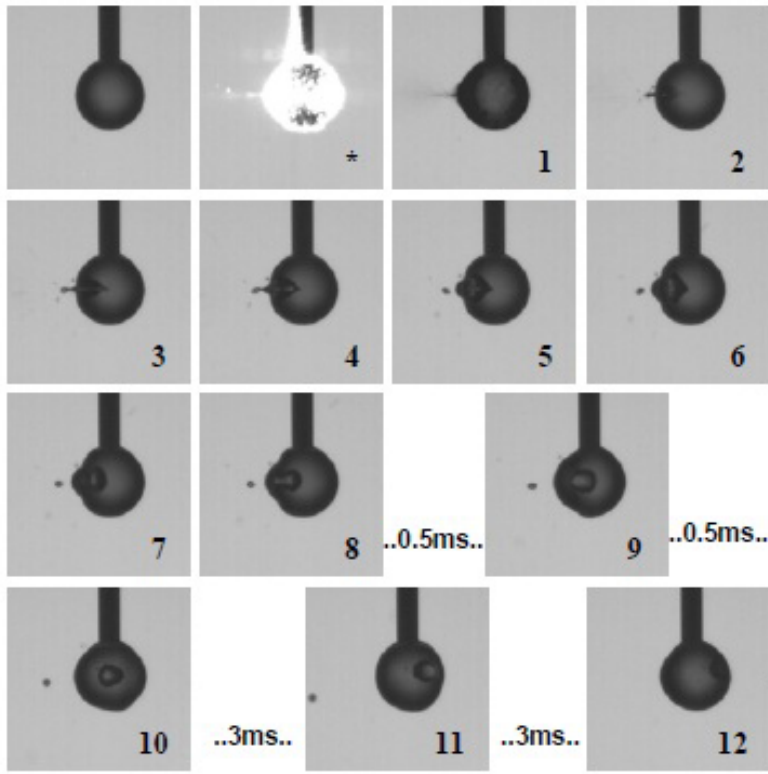

FIC. 30 Effects produced at resonant interaction on the droplet containing R6C at $10^{-3} \mathrm{M}$ in distilled water by the laser beam sent in the droplet's equatorial plane focused on its front surface (position 2 in Figure 2). $E_{3}=0.7 \mathrm{~mJ}$ Movie-25 (4,9 MB).

low speed. Since the record is 2D one cannot identify the real direction or rather the exact direction along which the nanodroplet(s) move.

By increasing the beam energy to $0.7 \mathrm{~mJ}$ one obtains more important effects on the droplet, as shown in Figure 30.

The $532 \mathrm{~nm}$ beam gradually penetrates through successive layers in the droplet and produces several types of effects:

- fluorescence emission following the strong absorption of the photons by the R6G molecules. In [20] is shown that an average increase of the droplet temperature of about $3^{\circ} \mathrm{C}$ is produced after absorbing the beam at $532 \mathrm{~nm}$. This originates in the nonradiative transfers of the absorbed energy in the R6G molecules, within the excited singlet states, between singlet states and between singlet and triplet states [36]. Locally, along the optical beam path in the droplet, the nonradiative transfers may lead to a much higher increase in temperature that may produce gas bubbles as results from frames 7- 11 in Figure 30.

- formation of a fast moving jet at the front face of the droplet that propagates backward, and of an associated crown which propagates also backward and which is formed by, most probably, nanodroplets [frame $\left(^{*}\right)$ and 1 in Figure 30.]

- formation of conic jets at the front surface which propagate backward at a lower speed and generate from the tip larger nanodroplets (frames 2 -11 in Figure 30); the cone emission is accompanied by emissions of other nanodroplets which propagate in $4 \pi$ near the front face of the droplet.

- overall droplet's deformation that is damped in a few ms.

The laser beam does not penetrate the droplet, neither produces any effects on the back surface of it. This is the case of 


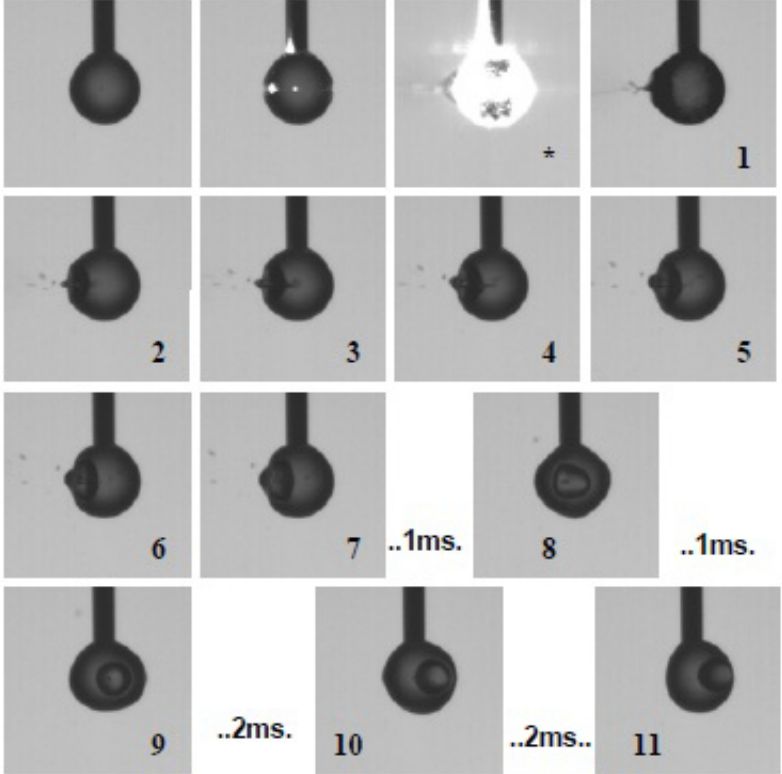

FIG. 31 Effects produced at resonant interaction on the droplet containing R6C at $10^{-3} \mathrm{M}$ in distilled water by the laser beam sent in the equatorial plane and focused on the front surface of the droplet (position 2 in Figure 2). $E_{4}=1 \mathrm{~mJ}$ Movie-26 $(4,9 \mathrm{MB}$ ).

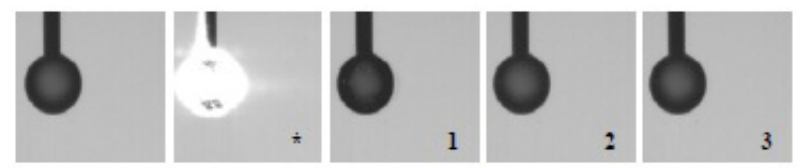

FIC. 32 Effects produced at resonant interaction on the droplet containing R6C at $10^{-3} \mathrm{M}$ in distilled water by the laser beam sent in the equatorial plane and focused on the back surface of it (position 3 in Figure 2). $E_{4}=1 \mathrm{~mJ}$ Movie-27 $(4,9 \mathrm{MB})$.

the interaction in the same geometry with a $1 \mathrm{~mJ}$ beam; the same kind of effects are produced on the droplet (Figure 31).

There are still some differences such as:

- the fast propagating jet at the front face is more consistent [frame $\left({ }^{*}\right)$ in Figure 31].

- the gas bubble/bubbles formed in the droplet have larger volumes/dimensions (frames 2-11 in Figure 31).

- the lower speed conic jet is associated with a crown (frame 1 in Figure 31) and the shape of this pair makes it practically identical with the same effect reported in [6] in the case of the interaction of a Nd:YAG laser beam with a hemispheric water drop emitted at $1.064 \mu \mathrm{m}$. Besides the experimental set-up differences between this work and [6], another difference is that in our experiments the effect is obtained at other wavelength, in an absorbing medium and at a power level of the laser beam that is about 20 times lower.

- the deformations of the droplet are larger.

If the focus is placed in the equatorial plane of the droplet on the back surface of it and the beam is perpendicular on the droplet surface, the typical effects onthe droplet are shown in Figure 32.
For all cases, the pumping beam is strongly absorbed at the input in the droplet and due to its energy density (Figure 3, Table 2) the radiation absorption in the droplet is not saturated . So, there are no available photons to apply pressure on the droplet and besides the intense fluorescence radiation no other effects - mechanical effects included - are produced on the droplet by the $532 \mathrm{~nm}$ beam.

\section{DISCUSSIONS / CONCLUSION}

The literature reports do not show too many data on the laser beams interactions with droplets in pendant/ suspended/hanging positions in open atmosphere. In dealing with this subject there are some experimental difficulties such as the contamination of the droplets content with impurities and their association with surface tension and contact angles variations, the vaporization of the droplets materials and the consequent modifications of their volumes, dimensions, shapes and components concentrations.

In this paper results regarding the unresonant and/or resonant interaction of droplets with pulsed laser beams focused on them are reported.

We consider that at interaction with a laser beam a distilled water droplet is an optical body, taking into account that $\mathrm{n}_{\text {water }}=1.33>\mathrm{n}_{\text {air }}=1.003$ at room temperature and at normal atmospheric pressure. In this way, the laser beam is refracted at the interface of the droplet with the surrounding air and may be internally reflected in the droplet up to the total reflection. Depending on its wavelength, the beam is either not absorbed in the droplet's material in the case of unresonant interaction or absorbed, at resonant interaction.

The interaction of the laser beam with a droplet is very fast, because the light needs about 8 ps to cross a distilled water droplet with diameter of around $2 \mathrm{~mm}$; it is so fast that one may consider that during the propagation in the droplet the light finds its material in a stable/frozen state from the fluidic effects point of view. All the effects produced on the droplet take place at later stages, except the emission of fluorescence radiation by the fluorophores in the droplet at resonant interaction, which takes place in a time interval comparable to the laser pulse FTWHM.

The focal length of the laser beam and its divergence at the impact with the droplet are very important in the interaction process. It seems that the longer the focal length and the lower the divergence in the interaction zone the more confined is the area around the focus which is directly affected by the beam and the lower the beam power needed to obtain light pressure effects on the droplet. This is also important since the errors in positioning the focus of the laser beam on the droplet at the front face, in the centre of it or on its back face might have a diminished influence on the quality of the interaction results. The position of the impact point with respect to the droplet's surface is important and may promote or not different kinds of light pressure effects.

Comparing the effects of the laser beam on the droplets at dif- 
ferent volumes, it seems that they are strongly dependent on the volume at the same pumping energy when focused in the centre of droplet. The smaller the volume, the more important is the emission of micro- and nano-droplets by the main droplet. Although the waist of the laser beam does not occur abruptly, the position of the focus with respect to the droplet surface is relatively critical and positioning errors even lower that $100 \mu \mathrm{m}$ may be important. Generally speaking the inertial effects are important in the behaviour of water droplets at unresonant interaction with the beam.

A velocity gradient of the water in the droplet is produced by interaction with the laser beam focused in the equatorial plane on its surface and due to the water viscosity. It increases with the increasing of the beam energy and is independent of the droplet's volume. The computed velocity gradients justify the measured expulsion speeds of water jets and micro-/nanodroplets emission immediately after the interaction with the laser beam. The viscosity parameters may influence the effects obtained on the droplets and the speeds of the material emitted out of them.

The indicated supersonic speeds are examples; they are not maximal values of such speeds that may be obtained by unresonant irradiation of water droplet with laser beams; some effects, particularly the electrostrictive unhomogeneities produced by focusing the beam at the centre of the droplet, might be produced above a threshold and it remains to detail this idea in future experiments.

The results reported on unresonant interaction show several effects on the distilled water droplet: damped deformations and vibrations, emissions of jets of liquids at supersonic speeds and of microdroplets and nanodroplets at high (supersonic) and low speeds, production of cylinder channels in the droplet that leads to formation of air bubbles inside it. The effects are of the same kind as those obtained by the application of electric or magnetic fields on dielectric fluids that are part of combinations of immiscible fluids.

The experiments have shown that the introduction of an obstacle in the droplet or at its surface - such as the capillary on which the droplet is suspended - may influence the type, quality and dimensions of the effects obtained at interaction with a laser beam.

The results on the resonant interaction have demonstrated that the effects of the laser beam on the droplet may be mixed with effects obtained at unresonant interaction and this depends on the laser beam energy or power density. It also depends on the droplet's volume and the absorbent properties and concentration in the droplet. One may have light absorption without having mechanical effects on the droplet because after absorption there are not available photons to build the pressure force. At higher beam energies, when there is an excess of photons in the droplet some effects specific to the unresonant interaction may be produced, nanojets emission included; regulating the absorption of the laser beam in the droplets (by varying the absorbent concentration for instance) may control the number and the characteristics of the effects produced by the light pressure.

\section{ACKNOWLEDGEMENTS}

This work was supported by the projects ANCS-PN2 41018/2007 and Nucleu LAPLAS 3, PN 09/2009. The authors thank Dr. S. Amarande, Dr. V. Damian, Dr. C. Lungu for helpful technical discussions and support.

\section{References}

[1] V. Nastasa, V. Pradines, I. R. Andrei, M. Boni, M. L. Pascu, and R. Miller, "Studies about the generation and characterisation of microdroplets with a controlled content," Optoelectron. Adv. Mat. 4 (11), 1916-1919 (2010).

[2] D. Psaltis, S. R. Quake, and C. H. Yang, "Developing optofluidic technology through the fusion of microfluidics and optics," Nature 442, 381-386 (2006).

[3] V. R. Horowitz, D. D. Awschalom, and S. Pennathur, "Optofluidics: field or technique?," Lab Chip 8, 1856-1863 (2008).

[4] M. L. Pascu, I. R. Andrei, M. Ferrari, A. Staicu, A. Smarandache, A. Mahamoud, V. Nastasa, and L. Liggieri, "Laser beams resonant interaction with micro-droplets which have a controlled content," Colloid. Surface. A 365 (1-3), 83-88 (2010).

[5] L. Rosenfeld, 0. M. Lavrenteva, and A. Nir, "Thermocapillary motion of hybrid drops," Phys. Fluids 20, 072102 (2008).

[6] S. T. Thoroddsen, K. Takehara, T. G. Etoh, and C.-D. Ohl, "Spray and microjets produced by focusing a laser pulse into a hemispherical drop," Phys. Fluids 21, 112101 (2009).

[7] J.-P. Delville, M. R. de Saint Vincent, R. D. Schroll, H. Chraibi, B. Issenmann, R. Wunenburger, D. Lasseux, W. W. Zhang, and E. Brasselet, "Laser microfluidics: fluid actuation by light," J. Opt. A-Pure Appl. 0p. 11, 034015 (2009).

[8] H.-R. Jiang, and M. Sano, "Stretching single molecular DNA by temperature gradient," Appl. Phys. Lett. 91, 154104 (2007).

[9] Y. Utsunomiya, T. Kajiwara, T. Nishiyama, K. Nagayama, S. Kubota, and M. Nakahara, "Laser ablation of liquid surface in air induced by laser irradiation through liquid medium," Appl. Phys. A 101, 137-141 (2010).

[10] R. Sahay, C. J. Teo, and S. T. Thoroddsen, "Laser-induced onset of electrospinning," Phys. Rev. E 81, 035302R (2010).

[11] A. Braun, C. Kornmesser, and V. Beushausen, "Simultaneous spatial and spectral imaging of lasing droplets," J. Opt. Soc. Am. A 22 (9), 1772-1779 (2005).

[12] H. Chraibi, D. Lasseux, R. Wunenburger, E. Arquis, and J.-P. Delville, "Optohydrodynamics of soft fluid interfaces: Optical and viscous nonlinear effects," Eur. Phys. J. E, 32, 43-52 (2010).

[13] A. De Giacomo, M. Dell'Aglio, 0. De Pascale, and M. Capitelli, "Spectroscopic investigation of laser-water interaction beyond the breakdown threshold energy," Spectrochim. Acta B 62, 87-93 (2007).

[14] J.-P. Delville, Introduction to Optofluidics, Course at The Abdus Salam International Centre for Theoretical Physics, Summer School, 1 - 5 June 2009.

[15] F. M. Sogandares, and E. S. Fry, "Absorption spectrum 340-640 $\mathrm{nm}$ of pure water. I. Photothermal measurements," Appl. Optics 35 (33), 8699-8709 (1997).

[16] W. Scott Pegau, D. Gray, and J. R. V. Zaneveld, "Absorption and attenuation of visible and near-infrared light in water: dependence on temperature and salinity," Appl. Optics 36 (24), 6035- 
6046 (1997).

[17] B. J. Kirby Micro- and nanoscale gluid mechanics: transport in microfluidic devices (Cambridge University Press, Cambridge, 2010).

[18] A. Ashkin, and J. M. Dziedzic, "Radiation pressure on a free liquid surface," Phys. Rev. Lett. 30, 139-142 (1973).

[19] R. Wunenburger, B. Issenmann, E. Brasselet, C. Loussert, V. Hourtane, and J.-P. Delville, "Fluid flows driven by light scattering," J. Fluid. Mech. 666, 273-307 (2011).

[20] M. L. Pascu, A. Smarandache, M. Boni, J. Kristiansen, V. Nastasa, and I. R. Andrei, "Spectral properties of some molecular solutions," Rom. Rep. Phys. 36, 1267-1284 (2011).

[21] D. R. Alexander, J. P. Barton, S. A. Schaub, and G. M. Holtmejer, "Nonlinear interaction of $\mathrm{KrF}$ laser radiation with small water droplets," Appl. Optics 30 (12), 1455-1460 (1991).

[22] Y. Utsunomiya, T. Kajiwara, T. Nishiyama, K. Nagayama, and S. Kubota, "Pulse laser ablation at water-air interface," Appl. Phys. A 99, 641-649 (2010).

[23] S. K. Y. Tang , R. Derda, Q. Quan, M. Loncar, and G. M. Whitesides, "Continuously tunable microdroplet-laser in a microfluidic channel," Opt. Express 19 (1), 135368 (2011).

[24] H.-M. Tzeng, K. F. Wall, M. B. Long, and R. K. Chang "Laser emission from individual droplets at wavelengths corresponding to morphology-dependent resonances," Opt. Lett. 9 (11), 499-501 (1984).

[25] J. Z. Zhang, and R. K. Chang, "Shape distorsion of a single water droplet by laser-induced electrostriction," Opt. Lett. 13, 916-918 (1988).

[26] S. M. Chitanvis, "Acoustic instability induced in compressible, transparent fluids by electrostrictive effects," Opt. Lett. 15 (14), 763-765, (1990).
[27] R. W. Boyd Nonlinear optics (Third Edition, Academic Press, London, 2008).

[28] C. C. Mei, Lecture Notes on Fluid Dynamics, MIT, in 1.631/2.21] Advanced Fluid Dynamics of the Environment, Course, Instructor T.R. Akylas (2007).

[29] V. L. Streeter, E. B. Wylie, K. B. Bedford Fluid Mechanics (McGrawHill, New York, 1998).

[30] F. F. Träger, Handbook of Lasers and Optics (Springer Science + Business Media, New York, 2007).

[31] P. T. Rakich, P. Davids, and Z. Wang, "Tailoring optical forces in waveguides through radiation pressure and electrostrictive forces," Opt. Express 18(14), 14439-14453 (2011).

[32] T. N. Buch, W. B. Pardo, J. A. Walkenstein, M. Monti, and E. Rosa, "Experimental issues in the observation on water drops dynamics," Phys. Lett. A248, 353-358 (1998).

[33] Y. M. Shin, M. M. Hohman, M. P. Brenner, and G. C. Rutledge, "Electrospinning: A whipping fluid jet generates submicron polymer fibers," Appl. Phys. Lett. 78 (8), 1149-1151 (2001).

[34] I. G. Loscertales, A. Barrero, I. Guerrero, R. Cortijo, M. Marquez, and A. M.Hanan-Calvo, "Micro/nano encapsulation via electrified coaxial liquid jets, " Science 295, 1695-1698 (2002).

[35] F. P. Schäfer, Dye lasers (Third Ed., Springer Verlag, New York, 1990).

[36] L. Danaila, M. L. Pascu Lasers in neurosurgery (Editura Academiei, Bucharest, 2001). 